\title{
Destinée des produits technologiques remplacés : l'influence de la valeur résiduelle perçue
}

\author{
Dominique Kréziak
}

Université Savoie Mont Blanc, IAE, IREGE, France

\section{Isabelle Prim-Allaz}

Coactis EA 4I6I, Université Lumière Lyon 2, France

\section{Elisabeth Robinot}

Ecole des sciences de la gestion de I'Université du Québec à Montréal (ESG- UQAM), Canada

\begin{abstract}
Résumé
L'accélération du rythme de remplacement des produits technologiques conduit à la mise au rebut de nombreux objets encore en état de fonctionnement. Dans une perspective durable d'économie circulaire, l'objectif de cet article est d'éclairer les destinées des objets technologiques remplacés, en s'appuyant sur leur valeur résiduelle perçue (VRP), définie comme la valeur que leurs propriétaires leur accordent alors qu'ils n'en ont plus l'usage. Dans un contexte de post-usage et de séparation avec les possessions, la VRP permet de prendre en compte simultanément un individu, un objet spécifique et une situation particulière, et d'estimer son influence sur la destinée de l'objet en question. Un outil de mesure de la VRP est développé autour de trois dimensions (utilitaire, financière et affective). Les résultats de régressions logistiques binomiales et multinomiales réalisées sur un échantillon de 1302 répondants, représentatif de la population française, montrent que la VRP explique bien le fait de garder ou non un produit remplacé et constitue un déterminant majeur du choix de filière lorsque cet objet n'est pas gardé. Des implications managériales en sont tirées, montrant comment la VRP constitue un levier pour favoriser la recirculation de l'objet et contribuant à résoudre les problématiques environnementales liées au gaspillage des ressources naturelles.
\end{abstract}

\section{Mots-clés}

destinées des objets, économie circulaire, tendance à tout garder, valeur post-usage, valeur résiduelle perçue

L'accélération du renouvellement des produits technologiques, souvent avant leur fin de vie technique, est un véritable enjeu économique, sociétal et environnemental, conduisant à une production accrue de déchets et au gaspillage de ressources rares. Si le remplacement anticipé des 
produits par leurs utilisateurs est souvent décrit dans les médias comme étant provoqué par les marques (les fabricants produiraient des biens à durée de vie limitée, dont l'obsolescence serait programmée), les consommateurs ont leur part de responsabilité.

A titre d'exemple, la durée d'utilisation moyenne des téléphones portables, par un acquéreur, serait selon une étude menée par l'association « les Amis de la Terre » en $2012^{1}$ de 18 mois, alors que leur durée de vie technique serait bien supérieure. Il semble dès lors indispensable de s'interroger sur la façon de prolonger la vie de ces objets technologiques, notamment par la promotion de destinées « responsables » offertes aux appareils remplacés.

Une étude récente publiée par l'Ademe (2017) indique que $88 \%$ des téléphones remplacés fonctionnaient encore au moment de leur remplacement. Le rapport Blandin (2016) indique que la majorité des téléphones remplacés sont ensuite gardés, au lieu de se voir offrir une deuxième vie. Celle-ci peut s'opérer soit sous forme de recirculation de l'objet soit sous forme de recyclage efficace des matériaux rares que contiennent ces objets. L'Ademe (2016) publiait également un rapport soulignant que la quantité des déchets ménagers aurait doublé au cours des 40 dernières années. Par ailleurs, ce même rapport indique que parmi les produits que les individus cessent d'utiliser, $15 \%$ sont des déchets électriques et électroniques, et représentaient en 2012 l'équivalent de 1,5 million de tonnes. Même si depuis une dizaine d'années, une grande partie de ce volume de déchet est trié, recyclé, apporté dans les déchèteries lorsque cela le nécessite, il reste toujours une partie importante qui est soit jetée, soit stockée dans les foyers et non valorisée, représentant ainsi un gisement de matières inexploité et dépourvu de toute valorisation possible.

Au-delà du rythme et des raisons du remplacement, et quel que soit leur état de fonctionnement au moment du remplacement, la question de la destinée des produits technologiques remplacés apparait donc cruciale dans une démarche de durabilité. Quelles sont les destinées que leur réservent leurs propriétaires et comment les expliquer ? Ainsi le sujet général de cet article est de comprendre pourquoi et comment les individus choisissent, après usage et remplacement, de garder ou non les objets, dont beaucoup sont encore en état de fonctionnement. Cette compréhension permettra de répondre à un enjeu sociétal et managérial majeur : améliorer la recirculation des objets, notamment celle des objets électroniques.

Pour ce faire, il est nécessaire de comprendre ce qui influence la destinée des produits technologiques remplacés (Jacoby et al., 1977). Au fil du temps, les recherches en comportement du consommateur ont élargi leurs perspectives pour ne plus se limiter seulement à la compréhension de l'acte d'achat mais intégrer également l'expérience de possession et d'utilisation des produits et services (Holbrook et Hirschmann, 1982). Toutefois, la phase d'élimination a reçu nettement moins d'attention que les deux autres phases.

La littérature décrit les destinées d'objets en fin de vie, identifie des trajectoires d'objets (Ertz et al., 2017 ; Kréziak et al., 2016), voire des profils d'individus, explore les motivations et les manières de faire pour se séparer des possessions, et explique en quoi ces décisions sont loin d'être seulement routinières et anodines (Roster, 2001), et ce même pour des objets usuels. La littérature montre, notamment, que cela varie selon les personnes (e.g. Coulter et Ligas, 2003 ; Guillard et Pinson, 2012 ; Haws et al., 2012), selon les objets considérés (e.g. Bianchi et Birtwistle, 2010 ; Cruz-Cárdenas et al., 2015 ; Phillips et Sego, 2011 ) et selon les circonstances (Ballantine et Creery, 2010 ; Cherrier, 2009 ; Cherrier et Murray, 2007 ; Cherrier et Ponnor, 2010 ; Kates, 2001 ; Kozinets, 2002 ; McAlexander, 1991 ; Pavia, 1993 ; Price et al., 2000 ; Roster, 2001 ; Young et Wallendorf, 1989). Mais, à notre connaissance, aucune recherche ne permet de considérer ces trois dimensions globalement. Dans cet objectif, nous mobilisons la notion de la valeur résiduelle perçue (VRP) centrée, pour le consommateur, sur la phase post-usage. Elle est définie comme étant la valeur que les individus accordent à un objet après usage. Ainsi, la valeur résiduelle perçue s'inscrit dans une vision interactionniste et relativiste de la valeur pour le consommateur (Rivière et Mencarelli, 2012) telle que proposée par Holbrook (1999) : une interaction 
entre un individu et un objet, relative car liée au contexte. Cette perspective invite donc à considérer la valeur perçue issue de l'interaction d'un individu avec un objet spécifique, dans une situation précise.

Une première partie est consacrée à la revue de littérature sur la fin de vie des objets et leurs destinées et mène, dans un second temps, à la proposition d'un cadre conceptuel centré sur la VRP. Celui-ci conduit à la formulation d'hypothèses sur les effets de la VRP sur la destinée des produits remplacés. Les choix méthodologiques et les instruments de mesure sont ensuite détaillés. Pour tester le modèle, un échantillon représentatif de la population française de 1302 répondants a été constitué. Les résultats montrent que la valeur résiduelle perçue est un déterminant majeur de la destinée des produits remplacés. En cas de non conservation de l'objet, la valeur résiduelle perçue explique encore mieux le choix de filière que le fait de ne pas le garder. Ces résultats conduisent à proposer des préconisations managériales pour favoriser la recirculation des objets technologiques remplacés.

\section{Mieux comprendre la fin de vie des objets}

Moins étudiée que les autres phases du processus de consommation, la fin de vie des objets constitue pourtant un champ à part entière de l'étude du comportement des consommateurs. Notons que le terme de fin de vie est utilisé ici et dans la littérature de façon globale, mais il recouvre en fait des réalités multiples. Il peut s'agir de la fin définitive de l'utilisation de l'objet, mais également la fin du temps de possession ou d'usage d'un bien, lorsque le possesseur se pose la question de le mettre au rebut ou lorsque le temps de la séparation éventuelle arrive. Ainsi, « fin de vie » peut être entendu au sens de la fin du cycle de vie « acquisition-utilisationélimination » (Jacoby et al., 1977). Toutefois, la perspective de l'économie circulaire incite à ne pas considérer qu'il s'agit de la fin de vie du produit, mais que celui-ci peut recirculer, en l'état, dans sa fonction première, ou en tant que matériau.

Suite aux premières typologies des comportements associés aux souhaits de se débarrasser d'un objet (Jacoby et al., 1977 ; Pieters, 1991), l'intérêt pour le sujet se développe lentement avec, cependant, une notable accélération ces dernières années (Monnot et Roux, 2017). En dépit des apparences, la littérature montre que se débarrasser ou se séparer de possessions n'est pas toujours un acte routinier et irréfléchi (Roster, 2001). Ainsi la décision de jeter ou de garder les objets peut impliquer des délibérations complexes et longues, même pour des objets courants (Roster, 2001), et ce d'autant plus que les symboliques attachées aux objets sont riches (Douglas, 1967) et que ceux-ci sont chargés d'identité (Kleine et al., 1995). Se séparer ou envisager de se séparer de ses possessions, même volontairement, les évaluer dans cette perspective, chercher la bonne personne à qui les transmettre (Lastovicka et Fernandez, 2005), décider de les garder ou de s'en séparer, et le cas échéant trouver une façon adéquate de le faire, s'interroger sur leur caractère identitaire et sur leur correspondance éventuelle avec le soi, sont autant de raisons de rendre cette étape de consommation complexe.

En effet, cette séparation peut renvoyer aux liens entre l'identité et le soi (Kleine et al., 1995). Young et Wallendorf (1989) parlent d'un « processus de détachement du soi » (process of detachment from the self). Même des objets de consommation courante, comme des tasses en carton ou des emballages, peuvent être perçus comme liés à l'identité de leur propriétaire et conduire après usage à une délibération élaborée, faisant en sorte qu'ils soient soigneusement recyclés plutôt que jetés (Trudel et al., 2016). La complexité du processus de séparation se reflète ainsi dans le temps nécessaire à le réaliser. Il peut être long et inclure une mise à distance spatiale et symbolique de l'objet dans des lieux de 'refroidissement' (Hirschman et al., 2012 ; McCracken, 1988), dans des rituels de transmission de possessions significatives (Lastovicka et Fernandez, 2005), via des stratégies pour garder le souvenir de l'objet (Winterich et al., 2017), ou encore par des efforts pour trouver un nouveau "foyer » aux anciennes possessions (Brough et Isaac, 2012). Se séparer d'objets peut aussi avoir un sens symbolique et constituer une forme de catharsis identitaire lors d'une transition subie ou choisie (Ballantine et Creery, 2010 ; Cherrier, 2009 ; Cherrier et Murray, 2007 ; Cherrier et Ponnor, 2010 ; Kozinets, 2002 ; McAlexander, 1991). Enfin, Türe (2014) montre 
que la perspective de se séparer d'une possession est utilisée comme l'ultime occasion d'augmenter encore la valeur globale retirée d'un objet, en choisissant soigneusement ce qui en sera fait. Lorsqu'ils sont gardés, ces objets peuvent donner lieu à différentes pratiques de réutilisation (e.g. Buckley, 2009), de réparation (e.g. Gregson et al., 2007), de transformation créative, d'upcycling ou de stockage (Cova et Kréziak, 2013). Parfois, l'individu garde simplement un objet faute d'avoir trouvé la filière adéquate pour s'en débarrasser (Türe, 2014).

En cas de séparation d'avec l'objet, les manières de faire (De Certeau, 1980) ne se limitent pas à jeter ou à recycler. Les destinées sont bien plus nombreuses et variées. Le dépôt aux encombrants peut se faire avec l'espoir que l'objet sera «adopté » par d'autres (Roux et Guillard, 2016). La pratique du don intègre une relation sociale entre un donneur et un receveur (Bergadaà, 2006 ; Roux et Guillard, 2016 ; Robinot et Trespeuch, 2016), que le don soit caritatif ou fait à l'entourage, en direct ou par internet (Bianchi et Birtwistle, 2010 ; Birtwistle et Moore, 2007 ; Domina et Koch, 1999 ; Ha-Brookshire et Hodges, 2009), ou encore en offrant un cadeau que l'on a soi-même reçu (Ertimur et al., 2015). La revente est aussi mobilisée (Albinsson et Perera, 2009 ; Denegri-Knott et Molesworth, 2009 ; Roux et Guiot, 2008), à travers de multiples canaux parfois soigneusement choisis, impliquant ou non la rencontre entre le vendeur et l'acheteur (Bianchi et Birtwistle, 2010 ; Morgan et Birtwistle, 2009). Cependant, ces pratiques ont souvent été abordées de façon éclatée dans la littérature. Or, il semble que l'étude de l'ensemble de ces destinées de façon simultanée renforcerait la compréhension du devenir des objets lorsque l'individu n'en a plus l'utilité (De Ferran et Robinot, 2012 ; 2013 ; Robinot et Trespeuch, 2016).

La littérature montre que la perspective et les modes de séparation des objets post-usage varient selon les individus, les objets et les situations. La façon dont la fin de vie des objets est envisagée oppose clairement la catégorie des « jeteurs » à celle des « gardeurs » (Coulter et Ligas, 2003 ; Guillard et Pinson, 2012 ; Haws et al., 2012). Cette opposition a notamment été étudiée au travers d'un trait de personnalité, la tendance à tout garder (Guillard et
Pinson, 2012 ; Haws et al., 2012). Le type, la nature et le caractère identitaire des objets jouent également un rôle important dans le processus (e.g. Bianchi et Birtwistle, 2010 ; Cruz-Cárdenas et al., 2015 ; Phillips et Sego, 2011 ; Trudel et al., 2016). Enfin, l'influence des circonstances ou de la situation est primordiale sur la perspective de se séparer d'objets. Le remplacement d'un produit peut aussi constituer une occasion particulière de délibérer sur le fait de le garder ou de s'en séparer (Cooper, 2004). De même, un déménagement (Desjeux et al., 1998), mais aussi les transitions de vie (Ballantine et Creery, 2010 ; Cherrier, 2009 ; Cherrier et Murray, 2007 ; Cherrier et Ponnor, 2010 ; Kates, 2001 ; Kozinets, 2002 ; McAlexander, 1991 ; Parsons, 2008 ; Pavia, 1993 ; Price et al., 2000 ; Roster, 2001 ; Young et Wallendorf, 1989) constituent des occasions de s'interroger sur le fait de garder ou non des possessions dont on n'a plus l'usage.

Peu de recherches, à notre connaissance, permettent de rendre compte de ces éléments de façon simultanée, intégrant un individu, un objet spécifique et dans une situation donnée. L'objet du présent travail est de répondre à ce manque en mobilisant la valeur résiduelle perçue, dans une vision interactionniste et relativiste de la valeur (Holbrook, 1999 ; Rivière et Mencarelli, 2012), qui permet simultanément d'intégrer ces trois aspects. En effet, éclairer les choix individuels de destinées dans un contexte et pour un objet spécifique implique de considérer ces différents éléments, présents dans la littérature mais de façon séparée, chaque recherche se concentrant sur l'une d'entre eux, soit une catégorie de produit, comme Bianchi et Birtwistle (2010) pour les vêtements, soit une situation particulière, comme Kates (2001) relativement à la maladie, soit un type de personnalité, comme Coulter et Ligas (2003) pour les gardeurs et non gardeurs.

\section{La valeur résiduelle perçue et les destinées de l'objet remplacé}

Pour Holbrook et Hirschmann (1982), l'expérience de consommation ne saurait se résumer à l'acquisition des objets, mais inclut l'ensemble de l'expérience de consommation que les individus en 
retirent. L'attention accordée au processus de séparation, mis en évidence dans la littérature, conduit à estimer que l'objet post-usage n'est pas forcément perçu comme sans valeur. La valeur de consommation (Rivière et Mencarelli, 2012) est définie par Holbrook $\left(1996^{2}\right.$; 1999) comme une expérience interactive et relativiste de jugement : elle implique une évaluation d'une expérience de consommation, et concerne simultanément un individu, un objet et une situation. La valeur résiduelle perçue d'un objet peut se définir comme la valeur que les individus accordent à un objet spécifique après usage (Rivière et Mencarelli, 2012).

La valeur résiduelle perçue (VRP) correspond à la valeur attribuée à une possession lorsque son propriétaire n'en a plus l'usage. Il s'agit d'une valeur post-usage qui renvoie à la valeur que l'individu confère à l'objet alors qu'il ne l'utilise plus dans sa fonction première. Dans la littérature portant sur la valeur, la notion de valeur résiduelle n'est pas systématiquement abordée, et ce, même dans des travaux de synthèse (e.g., Venkatesh et Penaloza, 2014). Ainsi dans leur analyse, qui se veut globale, de la valeur sur le cycle de vie du produit, Karababa et Kjeldgaard (2014) désignent la valeur d'un objet comme dynamique, subjective et liée au contexte, mais la fin de vie est absente de leurs propos. Lorsque la valeur résiduelle perçue est abordée, il s'agit généralement de travaux relatifs à une vision dynamique de la valeur et liée au cycle de consommation du produit (Parasuraman et Grewal, 2000 ; Woodall, 2003). Ainsi, Woodall (2003) distingue quatre types de valeurs différentes qui se succèdent au cours de la vie du produit : (1) la valeur perçue de l'objet avant l'achat, (2) sa valeur perçue au moment de l'achat, (3) sa valeur postachat et finalement (4) sa valeur résiduelle (disposition value). Les critères permettant d'apprécier la valeur d'un objet évoluent au fil du temps et du cycle d'interactions entre l'individu et l'objet (Woodall, 2003). Si Woodall (2003) pointe l'intérêt de la valeur résiduelle dans l'inventaire des différents états de la valeur, il ne détaille pas pour autant le concept. Les valeurs d'acquisition, d'échange (Bagozzi, 1975) et d'usage (Holbrook, 1999) ont ainsi été beaucoup plus étudiées que la valeur résiduelle. De plus, comme le regrettent Parasuraman et Grewal (2000), il n'y a pas de mesure appropriée de la perception des consommateurs de la valeur post-usage, contrairement notamment aux phases d'achat et de transaction.

Dans une perspective d'arbitrage entre gains et sacrifices comme composants de la valeur (Zeithaml, 1988), la valeur résiduelle se situe dans la littérature plutôt du côté des sacrifices. Ainsi, Woodall (2003) considère le coût éventuel lié au fait de se débarrasser de l'objet. La logistique induite par le traitement des résidus (Monnot et al., 2014) peut s'interpréter en termes de coûts non monétaires (temps, effort), ainsi que de coûts psychologiques et relationnels liés aux délibérations potentielles autour de l'élimination des objets. Thompson (1979) et Parsons (2008) considèrent que l'objet mis au rebut n'a plus de valeur, à l'instar de Roster (2001) pour qui il est celui dont le consommateur cherche à se débarrasser. A l'inverse, une dimension de valeur résiduelle positive est présente chez Penalosa et Mish (2011), pour qui la valeur globale d'un produit tient aussi aux éléments relatifs à sa fin de vie possible. Parasuraman et Grewal (2000) parlent de "redemption value » ou de valeur de rachat, en plus de la valeur d'achat et de la valeur tirée de l'usage. Ces auteurs la définissent comme le bénéfice résiduel (prix du produit) au moment de la reprise ou de la fin de vie du produit. Comme pour Okada (2001), il s'agit alors d'une approche « comptable », indiquant l'espérance de valeur de reprise du produit. Ces approches restreignent souvent la valeur résiduelle à sa seule dimension financière. Pourtant, Türe (2014) montre que d'autres formes de valeur peuvent être présentes dans la perspective de se séparer d'un objet : par exemple, en envisageant et réalisant un don caritatif, le possesseur rajoute une valeur morale à sa consommation et à sa possession de l'objet et renforce ainsi sa position de domination morale sur des personnes qu'il estime moins favorisées.

Enfin, la valeur résiduelle perçue influence les choix de consommation et notamment d'achat, à travers la valeur anticipée de reprise (Parasuraman et Grewal, 2000). Elle contribue à la valeur globale perçue et influence in fine la fidélité des clients (Parasuraman et Grewal, 2000). Elle peut retarder les décisions de remplacement (Guillard et Pinson, 2012), notamment lorsque les individus sont 
réticents à produire des déchets (Bolton et Alba, 2012) ou lorsqu'ils réalisent des arbitrages mentaux pour évaluer le coût induit pour se débarrasser d'un produit remplacé (Woodal, 2003).

Concernant les effets sur les destinées post-usage des possessions, la notion de valeur résiduelle n'est pas toujours explicite. La littérature consacrée au processus de séparation et d'élimination des objets suggère, comme détaillé dans la section précédente, que les individus accordent de la valeur à leurs objets après usage, et qu'il est complexe de s'en séparer, même pour des objets anodins ou dont ils n'ont plus l'usage. Türe (2014) propose une exploration fine de la façon dont les individus retirent de la valeur des objets dont ils se séparent. Dans une approche réflexive, l'auteur définit la valeur de séparation (value-in-disposition) comme la façon dont les individus utilisent le processus et la perspective de séparation d'avec les objets afin d'augmenter la valeur globale retirée de leurs possessions. L'auteure montre que le choix soigneux et réfléchi du mode de séparation et d'élimination est une façon de retirer le maximum de valeur globale : la filière choisie est celle qui va correspondre le mieux au type et à la quantité de valeur résiduelle qu'ils perçoivent dans l'objet dont ils se séparent, et permettre de la concrétiser. Ce processus transforme les possessions en transférant et créant une nouvelle forme de valeur, qui est potentiellement transférable à travers le choix approprié de filière. Kréziak et al. (2016) identifient trois dimensions de la valeur résiduelle perçue : (1) utilitaire (si le produit est perçu comme pouvant encore servir) ; (2) financière (si le possesseur estime que le produit a une valeur de revente potentielle); (3) affective (si le produit est considéré comme un souvenir, collectif ou individuel). Cependant, si l'effet sur la destinée est suggéré, il n'est pas mesuré.

Globalement la valeur résiduelle perçue apparaît dans la littérature sur la valeur dans les approches dynamiques, où la valeur évolue et se transforme au fil du temps, passant de valeur d'acquisition, à valeur d'échange, d'usage puis de post-usage, que nous étudions ici. Toutefois, dans la littérature, cette dernière étape est très peu détaillée comparativement aux autres formes de valeur et elle n'est pas mesurée. Lorsqu'elle est étudiée à travers les pratiques de fin de vie ou de recirculation des objets la valeur résiduelle n'est pas toujours explicitement formulée, et ne prend pas en compte simultanément l'effet d'interaction entre l'individu, l'objet et la situation. Ses effets sur les destinées ne sont pas estimés. Par conséquent, l'objectif de cette recherche est de mesurer l'effet de la valeur résiduelle attribuée à un objet particulier sur sa destinée après remplacement.

$\mathrm{Au}$ moment de la décision concernant la conservation ou la séparation d'avec l'objet, le type de valeur résiduelle perçue peut avoir une influence sur la décision. Cova et Kréziak (2013) montrent ainsi que des objets dont l'individu n'a plus l'usage sont gardés parce que «ça peut toujours servir ». L'individu estime alors la valeur résiduelle perçue de l'objet, qui sera d'autant plus gardé que celle-ci est forte (voir Figure 1). Nous formulons donc l'hypothèse suivante :

H1a: Plus la valeur résiduelle perçue utilitaire de l'objet remplacé est forte, plus l'objet est gardé.

Parasuraman et Grewal (2000) montrent que la valeur anticipée de reprise d'un objet lors de son remplacement influence l'achat. De ce fait, à l'occasion d'un remplacement, un produit remplacé sera d'autant moins gardé que sa valeur résiduelle d'ordre financier sera élevée. Nous formulons l'hypothèse suivante :

$H 1 b$ : Plus la valeur résiduelle perçue financière de l'objet remplacé est forte, moins l'objet est gardé.

Le caractère identitaire des possessions peut rendre la séparation difficile (e.g. Lastovicka et Fernandez, 2005 ; Roster, 2001). L'individu est réticent à se séparer d'un objet dont il perçoit les liens avec sa personnalité (Winterich et al., 2017). Un objet dont la valeur résiduelle perçue affective est élevée sera ainsi d'autant plus gardé. Nous formulons l'hypothèse suivante :

H1c: Plus la valeur résiduelle perçue affective de l'objet remplacé est élevée, plus l'objet est gardé. 


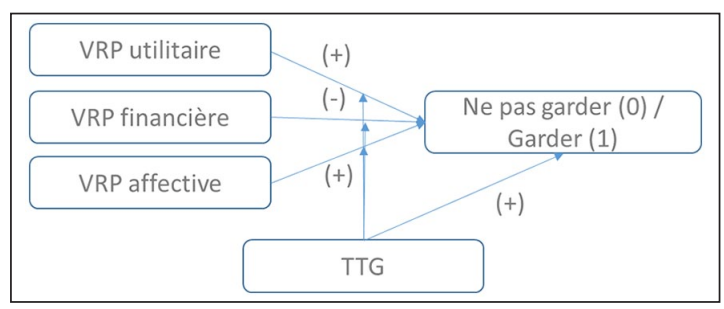

Figure I. Impact de la VRP sur la décision de garder ou de ne pas garder l'objet remplacé.

Suivant Türe (2014), nous considérons que le choix de la façon de se séparer d'un produit est une façon de maximiser la valeur globale retirée d'une possession. Le caractère transférable - ou non - de la valeur constitue un élément déterminant de choix de la destinée (Türe, 2014). Si l'objet n'est pas gardé, les choix de destinée des objets sont alors dépendants de la valeur résiduelle transférable, et sont envisagés comme une façon de réaliser leur valeur résiduelle perçue, c'est-à-dire de la rendre réelle et tangible. Dans cette perspective, les individus choisissent alors la façon qui va leur permettre d'optimiser la réalisation de cette valeur. Türe (2014) explique que le bon mode de séparation est celui qui compense le sacrifice lié à la séparation d'avec la possession, dans une logique de don et de contre don. Nous considérons donc qu'une filière sera choisie parce qu'elle permet de réaliser la valeur résiduelle perçue. Nous pouvons donc formuler la proposition générale selon laquelle le type de valeur résiduelle perçue influence le choix du mode de séparation. Plus spécifiquement, lorsque la valeur résiduelle perçue d'ordre utilitaire est élevée, et que l'objet n'est pas gardé, l'objet ne sera pas jeté pour autant. En effet, Bergadaà (2006) indique que pour une partie au moins des donneurs, l'objet donné est considéré comme une aide. C'est aussi le cas pour Albinsson et Perreira (2009) et Hibbert et al. (2005), pour le don ou la revente. Nous formulons, en conséquence, l'hypothèse suivante :

H2a : Lorsque l'objet remplacé n'est pas gardé, une valeur résiduelle perçue utilitaire forte conduit à le donner ou le revendre plutôt qu'à le recycler ou le jeter.
Gregson et Crewe (2003) et Denegri-Knott et Molesworth (2009) considèrent que les consommateurs, lorsqu'ils cherchent à se séparer d'un objet dont ils ne veulent plus, cherchent la meilleure façon de le faire : ils estiment sa valeur économique et sa valeur potentielle de revente. Denegri-Knott et Molesworth (2009) indiquent d'ailleurs que la perspective de revente sur ebay d'un produit, dont une personne n'a plus l'usage, réactive sa valeur économique, jusqu'alors parfois occultée. Nous formulons, en conséquence, l'hypothèse suivante :

$H 2 b$ : Lorsque l'objet remplacé n'est pas gardé, une valeur résiduelle perçue financière forte conduit à le revendre plutôt qu'à le donner, le recycler ou le jeter.

La dimension symbolique du don (Bergadàa, 2006), la volonté de transmettre le sens du produit et son identité, ainsi qu'une dimension de grandeur morale font partie des motivations pour donner un objet dont l'individu n'a plus l'usage (Türe, 2014). Le soin apporté à trouver un nouveau propriétaire pour les possessions faisant l'objet d'une valorisation affective ou liées à l'identité (Lastovicka et Fernandez, 2005 ; Trudel et al., 2016) permet de formuler l'hypothèse suivante :

$H 2 c$ : Lorsque l'objet remplacé n'est pas gardé, une valeur résiduelle perçue affective forte conduit à le donner plutôt qu'à le revendre, le recycler ou jeter.

Certains individus manifestent une tendance à tout garder -TTG - (Guillard et Pinson, 2012 ; Haws et al., 2012). Si Guillard et Pinson (2012) mesurent un effet direct de la TTG sur des comportements, ce trait de personnalité peut également modérer les effets de la valeur résiduelle perçue sur les destinées des produits, en augmentant l'effet de la valeur résiduelle perçue sur la décision de garder ou non un produit remplacé. Plus spécifiquement, une TTG forte peut renforcer l'effet de la VRP d'ordre utilitaire et affectif sur le fait de garder les produits remplacés, et réduire l'effet positif de la VRP 
d'ordre financière sur le fait de ne pas garder l'objet remplacé. L'effet quasi-modérateur de la TTG est ainsi testé3.

\section{Méthodologie}

Le choix du terrain d'application s'est porté sur le téléphone portable pour différentes raisons. Il s'agit d'un objet technologique dont le taux de pénétration est désormais très élevé, atteignant par exemple en France, en 2015, environ 92\% de la population totale - et $94 \%$ en 2017 dont $73 \%$ de smartphones ${ }^{4}$. Il est en forte évolution technologique, au cœur de nombreux lancements de la part des constructeurs qui proposent très régulièrement de nouvelles fonctionnalités. Il s'agit d'un petit objet à la fois facile à garder mais aussi à faire circuler. Le Ministère de la Transition Ecologique et Solidaire estime à 30 millions le nombre de téléphones inutilisés qui dorment dans les tiroirs des ménages français ${ }^{5}$ et souhaite favoriser la réparation, la revente, le recyclage ou encore le don pour donner une seconde vie à ces objets. Le jeter - à la poubelle - est ainsi considéré comme la destinée à éviter ${ }^{6}$.

Par ailleurs, un tel objet permet de constituer, avec des contraintes raisonnables, un échantillon de taille importante composé d'individus ayant réellement vécu un changement suffisamment récent, afin de limiter les effets de mémoire. Il est donc possible de demander aux répondants de se référer à la dernière fois qu'ils ont remplacé leur téléphone portable. Cette section méthodologique permet de présenter, dans un premier temps l'échantillon principal, puis dans un second temps les différentes échelles de mesure utilisées dans cette recherche. Les traitements des données sont ensuite exposés.

\section{Collecte des données}

Les données, utilisées pour tester le modèle, ont été collectées via un panel on line dans lequel les répondants sont récompensés par un système de cumul de points. Deux questions filtres étaient posées : (1) la première afin de savoir si les répondants avaient eu plusieurs téléphones (dans le cas contraire, ils étaient exclus de l'étude n'ayant pas été confrontés à la question du remplacement),
(2) la seconde pour s'assurer que leur dernier téléphone n'avait pas été volé ou perdu. Si tel était le cas, les répondants étaient également exclus de l'étude afin de n'avoir que ceux ayant été confrontés à la décision de la destinée de leur téléphone. De façon plus précise, les répondants étaient interrogés de la façon suivante : "Que diriez-vous de votre précédent téléphone au moment où vous l'avez changé ?». Le début du questionnaire positionnait le questionnement par rapport au dernier changement d'appareil. Il était bien spécifié : «Concernant votre dernier changement de téléphone portable... ». Ces instructions ont permis aux répondants de se réfèrer à une situation spécifique, la dernière fois où ils ont changé de téléphone portable, afin d'ancrer leurs réponses dans un contexte et un moment particulier qu'ils ont vécu.

Mille deux cent soixante-dix questionnaires ont ainsi été complétés dans un premier temps. Cet échantillon est représentatif de la population française des plus de 18 ans au regard de quatre critères : sexe, âge, revenu, répartition géographique ${ }^{7}$. L'âge moyen des répondants est de 41 ans (avec un minimum de 18 ans et un maximum de 66 ans écart type de 14 ans). Il est constitué de 636 femmes et 634 hommes (voir Tableau 1). Cependant, suite à la constitution de cet échantillon, 49 questionnaires supplémentaires ont été collectés par le cabinet d'études, via une question filtre pour ne retenir que des répondants ayant jeté leur ancien téléphone (voir Tableau 1). Pour maintenir la représentativité de l'échantillon, trente-deux répondants ont été retenus dans ce complément d'échantillon afin de pouvoir traiter ce comportement spécifique de façon statistiquement satisfaisante. En effet, si ce comportement est relativement marginal ${ }^{8}$, il est nécessaire d'améliorer sa compréhension dans le but de l'éviter (voir la Feuille de route pour l'Economie Circulaire publiée en avril 2018 par le Ministère de la Transition Ecologique et Solidaire ${ }^{9}$ ). Au final, les analyses de données visant à tester les propositions de recherche ont été réalisées sur un échantillon comprenant 1302 répondants, représentatif de la population française.

Afin d'éviter un effet d'ordre, les questions ont été présentées de façon aléatoire au sein de chaque bloc de questions (Carson, 1997). Cependant, pour respecter la logique du questionnaire, l'ordre des blocs est resté figé. 
Tableau I. Présentation de l'échantillon.

\begin{tabular}{|c|c|c|c|c|c|c|c|}
\hline & \multicolumn{3}{|c|}{ Echantillon principal $(n=1270)$} & \multicolumn{2}{|c|}{ Echantillon complémentaire $(n=32)$} & \multicolumn{2}{|c|}{ Echantillon total $(n=1302)$} \\
\hline & & $\mathrm{n}$ & $\%$ & $\mathrm{n}$ & $\%$ & $\mathrm{n}$ & $\%$ \\
\hline \multirow[t]{2}{*}{ Sexe } & Homme & 634 & 49,9 & 12 & 37,5 & 648 & 49,8 \\
\hline & Femme & 636 & 50,1 & 20 & 62,5 & 656 & 50,2 \\
\hline \multirow[t]{4}{*}{ Age } & Moyenne & \multicolumn{2}{|c|}{41 ans } & \multicolumn{2}{|c|}{36 ans } & \multicolumn{2}{|c|}{40,5 ans } \\
\hline & Minimum & \multicolumn{2}{|c|}{18 ans } & \multicolumn{2}{|c|}{19 ans } & \multicolumn{2}{|c|}{18 ans } \\
\hline & Maximum & \multicolumn{2}{|c|}{66 ans } & \multicolumn{2}{|c|}{58 ans } & \multicolumn{2}{|c|}{66 ans } \\
\hline & Ecart-type & \multicolumn{2}{|c|}{13,8 ans } & \multicolumn{2}{|c|}{9,8 ans } & \multicolumn{2}{|c|}{13,78 ans } \\
\hline
\end{tabular}

Tableau 2. Développement des mesures des construits.

\begin{tabular}{|c|c|c|c|}
\hline & Caractéristiques & & Objectifs du pré-test \\
\hline Echantillon I & $\begin{array}{l}\text { Téléphones portables, } \\
\text { Etudiants, } n=|5|\end{array}$ & $\begin{array}{l}75 \% \text { femmes, } 25 \% \text { hommes } \\
\text { Age moyen : } 22,39 \text { ans }(\min =20 ; \max =38 ; \\
\text { écart-type }=2,37)\end{array}$ & $\begin{array}{l}\text { Epuration des items } \\
\text { Structure factorielle }\end{array}$ \\
\hline Echantillon 2 & $\begin{array}{l}\text { Téléphones portables, } \\
\text { Adultes, } n=351\end{array}$ & $\begin{array}{l}60 \% \text { femmes, } 40 \% \text { hommes } \\
\text { Age moyen : } 43 \text { ans }(\min =18 ; \max =66 ; \\
\text { écart-type }=12,57)\end{array}$ & $\begin{array}{l}\text { Validation structure } \\
\text { factorielle }\end{array}$ \\
\hline Echantillon 3 & $\begin{array}{l}\text { Sèche-cheveux, } \\
\text { Adultes, } n=210\end{array}$ & $\begin{array}{l}42 \% \text { femmes, } 58 \% \text { hommes } \\
\text { Age moyen }: 40 \text { ans }(\min =18 ; \max =66 ; \\
\text { écart-type }=12,55)\end{array}$ & Validité externe \\
\hline
\end{tabular}

En sus de cette collecte principale, trois collectes de données sur échantillons de convenance ont été effectuées afin de valider les échelles de mesure (voir infra et Tableau 2).

\section{Mesures des construits}

Cette recherche nécessite notamment le développement d'un outil de mesure de la valeur résiduelle perçue. Pour ce faire, trois échantillons ont été constitués (voir Tableau 2).

Des analyses en composante principale exploratoires (rotations Promax) ont été réalisées, sous SPSS 25.0. Elles respectent les critères usuels de factorisation (KMO, teste de sphéricité de Bartlett, qualité de représentation) - Annexe 1, Tableaux 7 à 9. Dans un second temps, et sur l'échantillon final, des analyses confirmatoires ont été réalisées sous PLS-PM (version 18.07) : les $\alpha$ de Cronbach et les valeurs de l'indice de fiabilité composite ont permis d'évaluer la fiabilité des échelles. La validité convergente et la validité discriminante sont examinées selon la procédure de Fornell et Larcker (1981). Tous les indicateurs confirment la fiabilité et la validité des échelles de mesure (Tableaux 10 à 12, en Annexe). En effet, tous les $\alpha$ sont supérieurs à ,700. Tous les indices de fiabilité composite sont supérieurs à , 700 (Chin, 2010). La variance moyenne extraite (AVE) est supérieure à ,500 pour toutes les dimensions, indiquant une bonne validité convergente. Enfin, la variance moyenne extraite (AVE) est systématiquement supérieure aux carrés des corrélations indiquant une bonne validité discriminante - Tableau 12, Annexe 3 - (Hair et al., 2016).

Mesure de la destinée. La mesure de la destinée relève de la déclaration par les répondants de leur comportement, relativement à leur avant-dernier appareil. Un ensemble de modalités, issu de travaux précédents, notamment de Kréziak et al. (2016), a été proposé aux répondants (voir Tableau 3). Ces comportements peuvent être catégorisés en " garder » ou « ne pas garder » l'appareil remplacé. Les 
Tableau 3. Destinée des téléphones remplacés.

\begin{tabular}{|lrr|}
\hline Garder & & \\
\hline & $\mathbf{7 0 0}$ & $\mathbf{5 3 , 7 6 \%}$ \\
\hline N'en a rien fait & 438 & $33,64 \%$ \\
S'en est servi en dépannage & 224 & $17,20 \%$ \\
L'a donné comme jouet à un enfant & 38 & $\mathbf{2 , 9 2 \%}$ \\
\hline Ne pas garder & $\mathbf{6 0 2}$ & $\mathbf{4 6 , 2 4 \%}$ \\
\hline L'a donné à son entourage & 206 & $15,82 \%$ \\
La revendu à un particulier & 119 & $9,14 \%$ \\
L'a emmené au recyclage & 85 & $6,53 \%$ \\
L'a remis à un opérateur contre réduction & 75 & $5,76 \%$ \\
L'a jeté à la poubelle & 56 & $4,30 \%$ \\
L'a remis gratuitement à son opérateur & 29 & $2,23 \%$ \\
L'a rendu à son employeur & 13 & $1,00 \%$ \\
L'a donné à une association caritative & 11 & $0,84 \%$ \\
Autre & 8 & $0,61 \%$ \\
\hline
\end{tabular}

comportements de conservation (" garder ») proposés étaient : (1) Je l'ai gardé chez moi et je n'en ai rien fait ; (2) Je l'ai gardé chez moi et il a servi en dépannage ; (3) Je l'ai donné comme jouet à un enfant ${ }^{10}$. Lorsque les individus n'ont pas gardé leur appareil, les comportements proposés étaient les suivants : (1) Je l'ai donné à quelqu'un de mon entourage ; (2) Je l'ai donné à une association caritative ; (3) Je l'ai jeté à la poubelle ; (4) Je l'ai emmené au recyclage ${ }^{11}$; (5) Je l'ai revendu à un particulier (en direct ou sur un site) ; (6) Il a été repris gratuitement par l'opérateur ; (7) Il a été repris par l'opérateur qui m'a fait une réduction sur le nouveau ; (8) Je l'ai rendu à mon employeur ${ }^{12}$; (9) Autre. Dans les traitements relatifs à la situation où les répondants n'ont pas gardé, les réponses des répondants ayant coché les modalités (2), (8) et (9) n'ont pu être conservées du fait de leur faible nombre ${ }^{13}$.

Mesure de la valeur résiduelle perçue. La mesure de la valeur résiduelle perçue a été développée sur la base des travaux de Kréziak et al., (2016). Vingt et un items représentatifs de la valeur résiduelle ont été générés à partir d'une étude qualitative pour représenter les trois dimensions - utilitaire, financière et affective. Après avis d'experts, dix-huit d'entre eux ont été soumis à une première analyse en composante principale exploratoire (réalisée sur un échantillon de 151 étudiants) afin d'épurer l'échelle. Au regard des critères habituels de factorisation (qualité de représentation, loadings, alphas de Cronbach), et après plusieurs itérations, cette première analyse conduit à conserver 9 items, répartis sur les trois dimensions attendues : VRP utilitaire, VRP financière et VRP affective (voir Tableau 7, Annexe 1).

La mesure de la valeur résiduelle perçue, ainsi réduite à 9 items, a fait ensuite l'objet d'un nouveau test sur un échantillon de convenance constitué de répondants adultes $(\mathrm{n}=351)$, de tous horizons, de façon à correspondre à la population de l'échantillon final. Les items se distribuent à nouveau sur les trois dimensions, tel qu'attendu (voir Tableau 7, Annexe 1).

Enfin, pour vérifier la validité externe de la mesure, un test a été réalisé sur un autre produit (sèche-cheveux) et ceci sur un échantillon de convenance différent constitué de répondants adultes $(\mathrm{n}=210)$. Ce produit de petite taille appartient, comme le téléphone portable, à la catégorie des équipements électriques et électroniques et donc aux mêmes problématiques de fin de vie en termes de déchets ${ }^{14}$. La même structure factorielle est obtenue, confirmant la validité externe de l'échelle (voir Tableau 9, Annexe 1).

Cette structure est confirmée en analyse confirmatoire sur l'échantillon final (voir Tableau 10, Annexe 2). Ainsi, les analyses menées montrent la validité externe de l'échelle (re-test sur un produit différent, les sèche-cheveux) et sa validité convergente.

Tendance à tout garder. Par ailleurs, l'échelle de tendance à tout garder a été reprise de Guillard et Pinson (2012). Elle montre de très bonnes qualités psychométriques (voir Tableau 11, Annexe 2). 
Tableau 4. Modèle global - Régression logistique binomiale - Garder ou ne pas garder!'

\begin{tabular}{|c|c|c|c|}
\hline & B & Sig. & $\operatorname{Exp}(B)$ \\
\hline VRP financière &,- 719 & 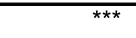 & ,487 \\
\hline VRP affective & 274 & *** & 1,315 \\
\hline TTG sentimentale &,- 230 & $* * *$ &, 795 \\
\hline TTG sociale &,- 325 & *** &, 722 \\
\hline TTG instrumentale &, 436 & *** & 1,547 \\
\hline TTG instrumentale ${ }^{*}$ VRP affective &,-127 & ** & ,881 \\
\hline Age* VRP utilitaire &, 006 & *** & 1,006 \\
\hline Implication * VRP utilitaire &,- 126 & ** & ,882 \\
\hline Constante & 230 & $\star \star \star *$ & 1,258 \\
\hline
\end{tabular}

'L'ensemble des tests de significativité relatifs aux régressions logistiques binomiales et multinomiales repose sur le test de Wald à un degré de liberté. Les valeurs de ce test ne sont pas reportées pour des raisons de lisibilité des tableaux. Seul le niveau de significativité apparaît.

Variables de contrôle. Afin de contrôler l'effet des caractéristiques individuelles, trois variables de contrôle ont été introduites dans le modèle : l'âge et le sexe des répondants ainsi que leur implication vis-à-vis de la catégorie de produits, dont la mesure est reprise de Strazzieri (1994).

\section{Résultats}

Du fait de la nature nominale des variables dépendantes retenues dans le modèle (comportements déclarés), des régressions logistiques binomiales et multinomiales ont été réalisées. Les hypothèses de recherche ont été testées sur les scores factoriels normalisés des construits issus des analyses confirmatoires précédemment présentées.

\section{Garder ou ne pas garder son téléphone}

L'impact des variables indépendantes sur la décision de garder ou ne pas garder son téléphone a été testé à l'aide d'un modèle global incluant les différentes dimensions de la VRP, de la TTG, ainsi que les effets d'interactions de la VRP avec la TTG et les autres variables de contrôle (implication envers la catégorie de produit, âge et sexe). Ce modèle global a été testé avec une régression logistique ascendante. Seules les variables ayant un effet significatif apparaissent dans la Tableau 4.
Le $\mathrm{r}^{2}$ de Nagelkerke du modèle est de 0,174 ( $\mathrm{p} \leq 0,001$ ) et l'AIC est de 1631,455 (Akaike, 1973). Ces résultats montrent que l'objet est d'autant plus gardé que sa VRP affective est élevée, et d'autant moins gardé que sa VRP financière est élevée (validant ainsi H1b et H1c). La VRP utilitaire n'a pas d'effet significatif au regard de l'ensemble de l'échantillon. H1a n'est donc pas validée. Cependant, la VRP utilitaire a un effet sur la décision de garder ou de ne pas garder au regard de l'âge : les plus âgés gardent un peu plus lorsque la VRP utilitaire est élevée. Elle a également un effet en fonction $\mathrm{du}$ niveau d'implication dans la catégorie de produit : les plus impliqués gardent un peu moins lorsque la VRP utilitaire est élevée. Les dimensions de la TTG exercent un effet direct sur la décision de garder ou de ne pas garder. L'objet est d'autant plus gardé que la TTG instrumentale du propriétaire (c'est-à-dire la tendance générale à considérer l'utilité des objets pour soi-même) est élevée, et d'autant moins gardé que la TTG sociale (c'est-à-dire la tendance à considérer l'utilité des objets pour les autres) est élevée. De façon plus surprenante, l'objet est moins gardé lorsque la TTG sentimentale (c'est-à-dire la tendance à considérer de façon générale le lien affectif aux objets) est plus élevée. La TTG instrumentale présente un effet d'interaction avec la VRP affective, lui conférant un caractère de quasi-modérateur dans la mesure où elle a également un effet direct. L'effet positif de la 
VRP affective sur le fait de garder est minoré dans le cas d'une forte TTG instrumentale.

\section{Choix de la filière}

L'impact des variables indépendantes sur le choix de filières, pour les individus n'ayant pas gardé leur téléphone, est testé au moyen de régressions logistiques multinomiales compte tenu de la nature de la variable dépendante (variable catégorielle à plus de 2 modalités). La catégorie de référence retenue est " jeter à la poubelle ", car celle-ci est considérée ici comme celle à éviter à tout prix. L'impact des variables indépendantes sur le choix de filière a été testé à l'aide d'un modèle global incluant les deux variables indépendantes (VRP et TTG) et les effets d'interactions de la VRP avec la TTG et les variables de contrôle (implication envers la catégorie de produit, âge et sexe) ${ }^{15}$. Ce modèle obtient un $\mathrm{r}^{2}$ de Nagelkerke de 0,577 et l'AIC est de 1451,524 (Akaike, 1973). Le choix de la filière est bien expliqué par les variables indépendantes retenues, et de façon notable par la VRP (voir Tableau 5). Les variables explicatives retenues expliquent nettement mieux le choix de filière $\left(\mathrm{r}^{2} \mathrm{de}\right.$ Nagelkerke $=0,577$ ) que le fait de garder ou de ne pas garder le produit remplacé $\left(\mathrm{r}^{2}\right.$ de Nagelkerke $=$ 0,174). Les trois dimensions de la VRP (utilitaire, financière et affective) de l'objet et la dimension instrumentale de la TTG de l'individu ont un effet significatif sur le choix de la filière. Toutefois, il n'y a pas d'effet d'interaction de la VRP avec la TTG, qui, par conséquent n'exerce pas d'effet de modération. Seule la variable âge présente un effet significatif d'interaction avec la VRP affective.

Plus spécifiquement, lorsqu'il n'est pas gardé, un produit remplacé aura d'autant plus de chances d'être donné à quelqu'un de l'entourage plutôt que d'être jeté lorsque la VRP utilitaire de l'objet est élevée. Ceci conduit à valider $\mathrm{H} 2 \mathrm{a}$. La revente ou le fait de faire reprendre gratuitement le téléphone par un opérateur seront également préférés au fait de jeter l'appareil. Les comportements de recyclage et de reprise par un opérateur contre réduction ne se distinguent pas du comportement de jeter sur cette dimension de la VRP.

Lorsqu'il n'est pas gardé et que la VRP financière de l'objet est élevée, un produit remplacé aura plus

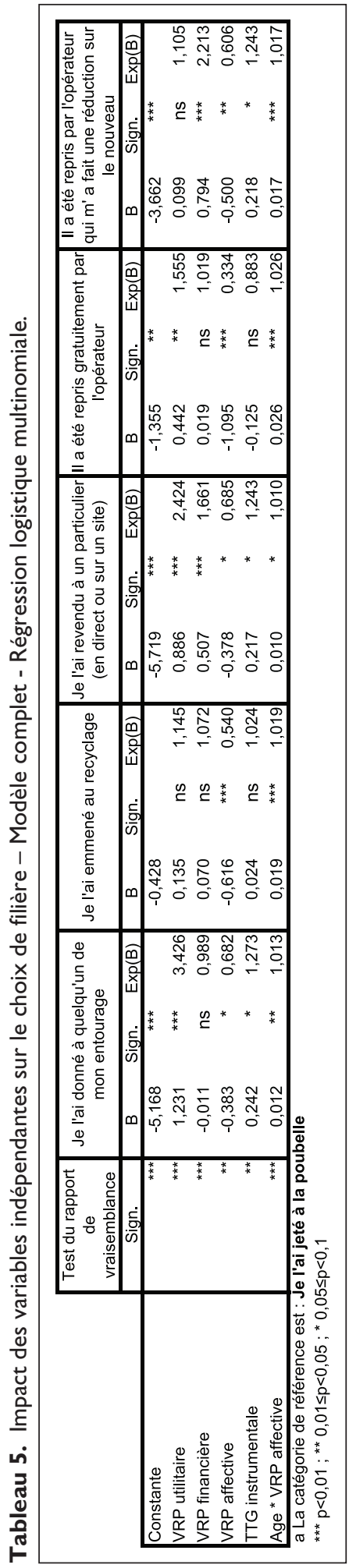


Tableau 6. Validation des hypothèses - Synthèse.

Hypothèse

Validation

$\mathrm{HIa}$ Plus la valeur résiduelle perçue utilitaire de l'objet remplacé est forte, plus l'objet est gardé.

Non validée

$\mathrm{HIb}$ Plus la valeur résiduelle perçue financière de l'objet remplacé est forte, moins l'objet est gardé.

Validée

$\mathrm{HIc}$ Plus la valeur résiduelle perçue affective de l'objet remplacé est élevée, plus l'objet est gardé.

Validée

$\mathrm{H} 2 \mathrm{a} \quad$ Lorsque l'objet remplacé n'est pas gardé, une valeur résiduelle perçue utilitaire forte conduit à

Validée

le donner ou le revendre plutôt qu'à le recycler ou le jeter.

$\mathrm{H} 2 \mathrm{~b}$ Lorsque l'objet remplacé n'est pas gardé, une valeur résiduelle perçue financière forte conduit à Validée le revendre plutôt qu'à le donner, le recycler ou le jeter.

$\mathrm{H} 2 \mathrm{c}$ Lorsque l'objet remplacé n'est pas gardé, la valeur résiduelle perçue affective forte conduit à le Non validée donner plutôt qu'à le revendre, le recycler ou jeter.

de chances d'être vendu ou rendu à un opérateur contre réduction plutôt que d'être jeté. Les comportements de jeter, donner et recycler ne se distinguent pas sur cette dimension de la VRP. L'hypothèse $\mathrm{H} 2 \mathrm{~b}$ est ainsi validée.

Enfin, lorsqu'un objet n'est pas gardé, il sera d'autant plus jeté que sa VRP affective est élevée. Ainsi, H2c n'est pas validée. La VRP affective distingue également le fait de jeter de toutes les autres modalités de destinée. Comme montré dans la section précédente, la VRP affective forte conduit plutôt à garder l'objet remplacé plutôt qu'à s'en séparer. Toutefois, si l'objet n'est pas gardé, une VRP affective forte augmente la probabilité que le produit soit jeté, plutôt qu'une pratique effective de recirculation.

L'âge a un rôle modérateur de l'effet de la VRP affective. Plus l'individu est âgé, plus la probabilité d'adopter le comportement de jeter est forte.

Concernant les effets de la TTG, seule sa dimension instrumentale a des effets significatifs. Lorsque l'objet n'est pas gardé, la TTG instrumentale augmente la probabilité de don, de revente ou de reprise contre réduction. Il n'y pas d'effet de modération de la TTG sur l'influence de la VRP.

\section{Discussion}

L'objet de cette recherche était de mettre en évidence, de définir et de mesurer le concept de valeur résiduelle perçue afin d'estimer ses effets sur les destinées des produits technologiques remplacés. La contribution de cette recherche se situe sur plusieurs plans, théorique, méthodologique et managérial.
Sur le plan théorique, cette recherche contribue à compléter la connaissance de la valeur perçue, en apportant des éléments théoriques sur la phase postusage, moins étudiée que les autres phases de la consommation. Le concept de valeur résiduelle perçue s'inscrit dans la lignée des travaux de Türe (2014) ainsi que dans la perspective dynamique de la valeur (Parasuraman et Grewal, 2000 ; Woodall, 2003).

La valeur résiduelle perçue peut être définie comme la valeur que les individus accordent à un objet après usage. Nos résultats valident empiriquement l'existence de trois dimensions financière, affective et utilitaire - proposées à partir de la littérature (Kréziak et al., 2016 ; Türe, 2014).

Cette recherche contribue également à la compréhension des facteurs qui influencent les destinées des produits remplacés (1) en considérant simultanément l'ensemble des destinées et, (2) en s'appuyant sur des comportements déclarés et non sur des intentions (voir Figure 2). Ainsi, la VRP contribue à expliquer le fait de garder ou non un objet. Dans le cas où il n'est pas gardé, la VRP influence le choix de la filière retenue. Elle contribue largement plus à expliquer ce choix de filière que le fait de garder ou de ne pas garder $\left(\mathrm{r}^{2}\right.$ de Nagelkerke $=0,577$ et 0,174 , respectivement). Les effets de la VRP sont différents selon les dimensions, soulignant la pertinence de les mesurer séparément et d'en évaluer l'influence de façon distincte, sans recourir à un score global qui pourrait être trompeur.

Plus spécifiquement, en intégrant la TTG comme variable quasi-modératrice et en contrôlant l'implication envers la catégorie de produit, l'âge et le sexe, les résultats mettent en évidence que les 


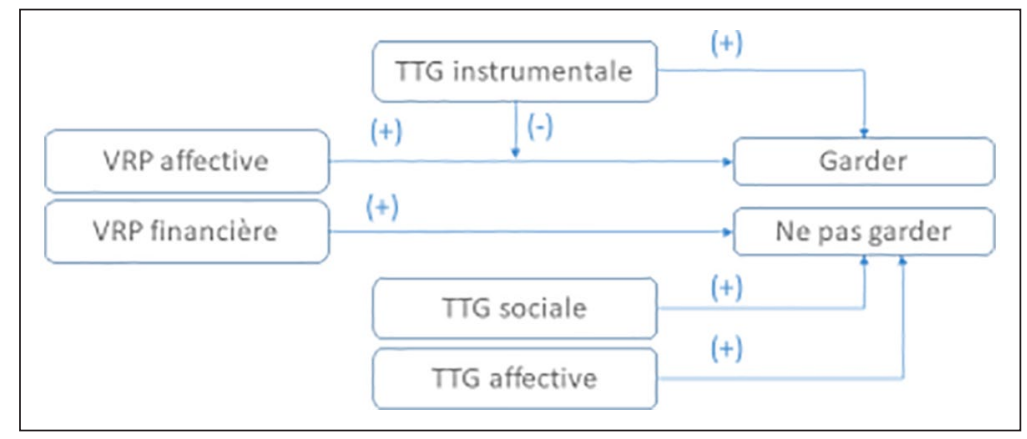

Figure 2. Synthèse des principaux effets conduisant à garder ou ne pas garder l'objet remplacé.

dimensions affective et financière de la VRP affectent la décision de garder ou ne pas garder l'objet. L'attachement à l'objet (Bowlby, 1979) est un frein à sa recirculation, et conduit à le conserver même après son remplacement. C'est d'ailleurs ce qui est fait dans la majorité des cas $(55 \%$ par les répondants de notre échantillon). Ceci peut être à relier à l'effet de dotation - endowment effect (Kahneman et al., 1991), où des individus préfèrent le statu quo (ici garder) au changement et demandent pour se séparer d'un produit qui leur appartient, un prix plus élevé que celui qu'ils sont prêts à payer pour l'acquérir. Ces individus auraient donc selon cet effet de dotation une aversion à la dépossession, considérant que le marché sous-évalue leurs possessions et préfèrent, en conséquence, garder. Nos résultats indiquent toutefois que la valeur résiduelle financière facilite la recirculation : si le prix de reprise ou de revente est suffisamment incitatif, c'est-à-dire si le marché estime le produit de façon satisfaisante, le produit peut recirculer. Enfin, la VRP utilitaire n'a pas d'influence directe sur cette décision. Le fait de penser que l'objet peut encore servir ne conduit pas à un comportement spécifique. Cependant, la VRP utilitaire joue sur la décision de garder ou de ne pas garder lorsque l'on prend en compte des variables modératrices. Un léger effet d'interaction est observé avec l'âge et le degré d'implication dans la catégorie de produit. L'effet de la VRP utilitaire est d'autant plus fort sur le fait de garder que l'individu est plus âgé, et d'autant plus faible que l'individu est faiblement impliqué. Cet effet d'interaction de l'âge, même s'il est faible, méritera d'être plus amplement investigué car contraire à la littérature sur la générativité qui tend à indiquer que les personnes les plus âgées transmettent pour prendre soin des générations futures (Urien et Kilbourne, 2011). Relativement à l'effet de l'implication, ce résultat pourrait être lié au fait que les individus les plus impliqués seraient plus tentés par un renouvellement rapide et ne peuvent stocker indéfiniment les anciens modèles (Helm et Conrad, 2015).

Trois des quatre dimensions de la TTG ont elles aussi un effet direct sur le fait de garder ou de ne pas garder l'objet remplacé (seule la dimension économique n'a pas d'effet). Comme cela pouvait être attendu, la dimension instrumentale de la TTG (le fait d'avoir tendance à garder les objets pour soi) favorise le fait de garder l'objet. A l'inverse, la dimension sociale favorise le fait de ne pas garder l'objet. Ce point s'explique assez aisément car cette dimension traduit le fait d'une tendance à garder « pour les autres ». Ainsi, l'objet quitte logiquement son propriétaire. Par ailleurs, l'objet sera moins gardé lorsque la dimension sentimentale de la TTG est élevée : cela pourrait signifier que ce produit n'est pas affecté par ce trait de personnalité, c'est-àdire que les personnes ayant tendance à tout garder ne sont pas spécialement attachées à leur téléphone ou qu'il n'entre pas dans les produits à garder pour ces raisons sentimentales. Cela montre cependant que la TTG sentimentale est un trait de personnalité que l'on ne peut confondre avec la VRP affective, spécifique à un produit et à une situation.

Les effets de la valeur résiduelle perçue ne sont pas les mêmes que ceux de la tendance à tout garder. Cela souligne plus globalement l'intérêt de mesurer la valeur résiduelle, pour un produit spécifique et dans une situation spécifique, dans une perspective 


\begin{tabular}{|c|c|c|}
\hline \multicolumn{2}{|c|}{ VRP utilitaire } & \multirow[b]{2}{*}{$\begin{array}{l}\text { - Revendre à un } \\
\text { particulier } \\
\text { - Rendre contre } \\
\text { réduction }\end{array}$} \\
\hline $\begin{array}{l}\text { - Rendre } \\
\text { gratuitement } \\
\text { - Revendre à un } \\
\text { particulier } \\
\text { - Rendre contre } \\
\text { réduction }\end{array}$ & plutôt que Jeter & \\
\hline \multicolumn{2}{|c|}{ VRP affective } & TTG instrumentale \\
\hline Jeter plutôt que & $\begin{array}{l}\text { - Recycler } \\
\text { - Donner } \\
\text { - Revendre à un } \\
\text { particulier } \\
\text { - Rendre contre } \\
\text { réduction }\end{array}$ & $\begin{array}{l}\text { - Donner } \\
\text { - Revendre à un } \\
\text { particulier } \\
\text { - Rendre contre plutôt que Jeter } \\
\text { réduction }\end{array}$ \\
\hline
\end{tabular}

Figure 3. Synthèse des éléments conduisant au choix de filière (comportement de référence : jeter l'objet).

interactionniste (Holbrook, 1999), indépendamment et de façon non superflue à l'estimation d'un trait général de personnalité. La diversité des effets des différentes dimensions de la TTG suggère l'intérêt d'effectuer des analyses au niveau des dimensions du construit pour estimer sa validité prédictive sur ce type de comportement, plutôt qu'en recourant à un score global (e.g. Guillard et Pinson, 2012).

Lorsque l'objet n'est pas gardé, la valeur résiduelle perçue influence le choix du mode de séparation. Nos résultats sont en cohérence avec Türe (2014) : la destinée choisie est celle qui correspond le mieux au type et à l'intensité de la valeur résiduelle perçue, celle qui la « réalise », comme pour une valeur en bourse. Plutôt que de jeter, une VRP utilitaire élevée favorise le don à l'entourage ; une VRP financière élevée favorise la revente ou le fait d'être repris par un opérateur contre réduction; la VRP affective joue un rôle dans le choix de la filière notamment entre le comportement de jeter versus emmener au recyclage. Une VRP affective forte accroît le comportement de jeter, soulevant un certain paradoxe de la part des propriétaires. Ainsi, une VRP affective forte conduit à garder le produit remplacé, mais si la séparation a lieu, tout sera fait pour éviter la recirculation du produit et ne pas partager cette valeur. Cet effet est renforcé par l'âge. Cela peut être interprété comme une rupture forcée du lien entre l'objet et son propriétaire, ou renvoyer aux résultats de Türe (2014), de Bergadaà (2006) ou de Brough et Isaac (2012) : Si le possesseur ne trouve pas la filière qui rend compte fidèlement de la valeur résiduelle affective importante attribuée au produit, ou qui semble moralement adéquate, il peut renoncer à s'en séparer et, si nécessaire, choisir une rupture brutale voire sacrificielle du lien, allant à l'encontre de ce que suggèrent les travaux sur la générativité mentionnés précédemment (Urien et Kilbourne, 2011).

Enfin, les effets comparés de la VRP et de la TTG montrent de nouveau que la VRP - au travers de ses trois dimensions - est distincte dans ses effets de la TTG, et qu'il est pertinent de l'estimer selon les situations et les objets (voir Figure 3).

Cette recherche contribue également à compléter la littérature sur la fin de vie des objets et sur leur recirculation en modifiant la perspective de leurs études. En effet, dans ce travail, l'accent est davantage mis sur l'objet en lui-même et la façon dont il est perçu, et moins sur la personnalité des individus et leur motivation (Bergadaà, 2006 ; Bianchi et Birtwistle, 2010 ; Denegri-Knott et 
Molesworth, 2009 ; Guillard et Pinson, 2012). Ce n'est plus seulement la corde altruiste qui est touchée, ou le besoin de faire du vide, la culpabilité d'avoir remplacé un objet, la sensibilité environnementale ou plus globalement la personnalité de l'individu qui sont au cœur, mais la relation à l'objet. Dans ce cadre, le ressort de l'attachement à chaque objet (Bowlby, 1979 ; Kleine et al., 1995 ; Robinot et al., 2014 ; Schifferstein et Zwartkruis-Pelgrim, 2008 ; Brezet et Van Hemel, 1997) et aux possessions peut influencer sa recirculation.

Nos résultats contribuent au champ de la recherche sur la valeur de consommation, envisagée dans sa perspective dynamique, où la valeur évolue au fil du temps (Rivière et Mencarelli, 2012 ; Woodal, 2003). Elle étend la connaissance de la phase post-usage, qui n'est parfois pas même pas citée par les auteurs (Karababa et Kjeldgaard, 2014 ; Venkatesh et Penaloza, 2014), ou seulement évoquée. Nuançant le point de vue de Okada (2001), Parasurama et Grewal (2000) et Roster (2001), nos résultats montrent que cette valeur existe et qu'elle ne se réduit pas à une valeur comptable liée à sa reprise, mais qu'elle intègre trois dimensions liées à sa valeur utilitaire, affective et financière. Enfin, nos résultats prolongent la contribution de Türe (2014) sur la valeur post-usage en en proposant une validation empirique. Un instrument de mesure a été développé, répondant à l'appel de Parasuraman et Grewal (2000). La contribution porte également sur la pertinence de la perspective interactionniste de la valeur (Holbrook, 1996), comme sur une de ses limites. Le pouvoir explicatif de la valeur résiduelle et de ses trois dimensions sur les destinées est significatif, et particulièrement important sur le choix de filière. En prenant en compte à la fois l'individu, le type de produit et la situation spécifique, les comportements sont bien expliqués, mais c'est toutefois au détriment du caractère généralisable des résultats, qui pourront être différents pour une autre situation ou un autre produit.

La contribution de cette recherche est également d'ordre méthodologique, avec la proposition d'un instrument de mesure de la valeur résiduelle perçue d'un produit remplacé, dont les qualités psychométriques sont satisfaisantes. L'instrument de mesure a été validé sur deux produits électronique (téléphone portable) et électrique (sèche-cheveux). En termes de validité discriminante, la valeur résiduelle perçue a été distinguée conceptuellement et empiriquement de la TTG. Enfin, sur le plan empirique, les relations ont été testées sur un échantillon de 1302 répondants représentatif de la population française, montrant la validité prédictive du construit.

Ces contributions se poursuivent sur le plan praxéologique. D'un point de vue managérial et dans une perspective d'économie circulaire, certaines destinées sont plus souhaitables que d'autres : l'enjeu managérial est donc de trouver les ressorts qui permettront d'améliorer les pratiques en termes de comportements souhaitables sur le plan environnemental et sociétal. La VRP constitue un levier pour favoriser la recirculation de l'objet. Lorsque le remplacement est acté, la valeur résiduelle perçue peut être utilisée comme un levier pour ne pas stocker son ancien appareil et choisir une filière permettant sa valorisation, adapté au produit et à la situation. Pour éviter les stocks inutiles de produits remplacés, la VRP, situationnelle et liée au type de produit, peut être actionnée plus facilement qu'un trait de personnalité comme la tendance à tout garder. La VRP influence tout particulièrement le choix de filière. Il s'agit d'éviter le stock mort d'appareils (i.e. favoriser le « ne pas garder »), en faisant expliciter au moment $\mathrm{du}$ remplacement la nature et l'intensité de la valeur résiduelle perçue de l'objet remplacé. La VRP financière conduit à se séparer de l'appareil : le calcul de la valeur de reprise peut être systématiquement fait. Si le résultat est suffisamment incitatif, il pourra aider à la revente. Sinon, ce calcul pourra servir de première étape dans un cycle d'engagement pour proposer une alternative (Beauvois et Joule, 1981). Mais c'est la VRP affective qui doit retenir l'attention, car elle incite à garder, et à jeter en cas de séparation. L'individu peut être alors conduit à exprimer ce qui fonde son attachement. Si c'est le contenu, une aide au transfert des données pourra être apportée sur le champ. Si c'est l'esthétique, une photo de type « souvenirs » pourra être proposée (Winterich et al., 2017). Si le produit fonctionne encore, la corde morale de l'aide à autrui et de la valorisation 
personnelle qu'elle peut apporter pourra être activée (Türe, 2014). Lors du choix de la filière adéquate, l'enjeu est encore différent. A chaque occasion de remplacement, l'individu peut être guidé pour déterminer la nature et l'intensité de la VRP du produit et pour identifier la filière la plus adaptée à cette VRP, c'est-à-dire celle qui permettra d'en retirer le maximum de valeur (Türe, 2014), l'objectif étant d'éviter absolument le comportement de « jeter », mais également le gaspillage que peut constituer le recyclage direct alors que l'appareil peut encore servir et circuler. Si la VRP utilitaire est forte, il est alors pertinent d'inciter à faire circuler l'objet : donner si la VRP financière est faible, revendre si elle est forte. Lorsque ces deux dimensions sont faibles, la valorisation par le recyclage est la plus adaptée, en évitant le comportement de jeter via une proposition immédiate de reprise en boutique, ou la recherche automatique du point de collecte le plus proche. La présentation de l'impact environnemental d'une telle action serait également un levier au choix de la filière la plus appropriée à la VRP. Une forme de nudge pourrait être imaginée à ce stade pour inciter à la bonne décision (Thaler et Sustein, 2008). Enfin, l'effet modérateur de l'âge suggère la possibilité d'un message différent pour certaines cibles plus âgées, soulignant le caractère génératif du fait de faire circuler le produit dont la VRP utilitaire est forte, alors que certains l'auraient plutôt gardé.

Pour étendre la réflexion, la collaboration des entreprises (fabricants et opérateurs) est nécessaire si l'on veut parvenir à une plus grande circulation et valorisation des objets technologiques remplacés. Aider le client à expliciter la VRP de son produit peut être une opportunité pour une marque d'accompagner son client sur toute la vie du produit dans une optique de qualité de service et de renforcement de la relation client, dans la perspective de créer de la valeur durable, persistante dans le temps, entre producteur et consommateur (Kumar et Reinartz, 2016). Les entreprises pourraient mettre à disposition des pièces de rechange, proposer des garanties de façon systématique, optimiser les possibilités de réparation en période de garantie, alléger les coûts de réparation pour les particuliers (en créant des modulations de garantie), faire en sorte que davantage de pièces détachées, de consommables ou de batteries le soient de manière abordable et, enfin, mettre à disposition des tutoriaux pour aider les consommateurs à réparer leurs objets.

\section{Limites et voies de recherche}

Des limites sont à signaler à ce travail, notamment sur le plan de sa mise en œuvre empirique. Elles constituent autant de voies de recherche et d'approfondissement. Si l'échelle de mesure de la VRP a été validée sur deux catégories de produits, les hypothèses n'ont, elles, été testées que sur une seule catégorie de produits. Il conviendra donc de répliquer les analyses sur d'autres produits technologiques afin de renforcer leur validité externe. Aussi, d'autres destinées pourraient émerger : ainsi la séparation temporaire d'avec les objets, en location ou prêt, a fait l'objet à notre connaissance de peu de travaux. Par ailleurs, le développement du prêt entre particuliers à travers des sites devrait susciter de nouvelles recherches dans ce sens.

Au-delà des réplications, la réflexion sur la VRP peut être étendue à tout matériau ou objet déchu, pas seulement aux objets technologiques. Ainsi l'absence d'effet de la dimension utilitaire de la VRP pourra être différente pour un autre type de produit : certains produits pourraient être davantage gardés à ce titre, alors que d'autres pourraient circuler davantage. Les effets d'interactions pourraient être explorés de façon plus approfondie, et notamment celui de l'âge, qui apparait ici comme un modérateur, mais pas seulement. Intégrer, au-delà de l'implication, le degré d'usage du produit concerné et sa durée de vie idéale perçue pourrait enrichir le modèle.

La notion même de VRP permet de sensibiliser au gaspillage potentiel que constitue le fait de jeter, mais aussi de recycler directement alors qu'un produit fonctionne encore ou peut être réutilisé, dans la même fonction ou autrement. Le discours est alors plus positif : " on réalise une valeur, on fait durer le produit même si on s'en sépare ». Pour d'autres produits, le recours à cette notion permet d'éliciter les destinées qui permettent de réaliser au mieux la valeur résiduelle perçue, l'inscrivant dans une démarche d'upcycling (Cova et Kréziak, 2013 ; 
Wilson, 2016). Dans cette perspective qui consiste à tirer le maximum de valeur de chaque unité de ressource naturelle extraite, et de chaque objet déchu, la valeur résiduelle perçue contribue à la réflexion sur l'économie circulaire (Cova et Kréziak, 2016).

Le choix de mesurer des comportements déclarés et non des intentions a contraint le type d'analyses réalisables. Toutefois mesurer des comportements et non pas des intentions futures a semblé plus pertinent sur le plan scientifique mais également managérial. En effet, certains auteurs (Carrington et al., 2010; Webb et Sheeran, 2006) soulignent que la mesure des intentions n'est pas un indicateur fiable des comportements. Les comportements mesurés ici sont déclarés, l'étape suivante serait de trouver une façon de partir de comportements réels. Dans tous les cas, la validité prédictive des modèles lorsque des comportements sont analysés est beaucoup plus fiable (Bemmaor, 1995 ; Chandon et al., 2005 ; Manski, 1990).

D'autres perspectives de recherche semblent prometteuses. Il serait pertinent de mesurer également la valeur d'usage au moment du remplacement, afin de la comparer explicitement avec la valeur résiduelle perçue, et d'éclairer la dynamique de cette métamorphose. Explorer le rapport à la possession et à la propriété et ses effets sur le remplacement des produits constitue une piste d'investigation, permettant de considérer autrement la fin de vie des objets, leur destinée, leur circulation, leurs valeurs et leurs usages, dans une perspective de servicisation des objets (Baines et al., 2007 ; Belk, 2013) et engageant les entreprises vers de nouvelles formes de modèles d'affaires (Ambroise et al., 2018).

\section{Remerciements}

Les auteures remercient l'ADEME pour le soutien financier apporté à cette recherche dans le cadre du projet COOP (Consommateurs et objets à obsolescence programmée) APR 2012, Déchets \& Société. Elles remercient également très sincèrement les trois relecteurs anonymes et les deux rédacteurs en chef successifs (Nil Özçaglar-Toulouse et David Gotteland) pour leurs commentaires avisés, qui ont largement contribué à la qualité de cette recherche, ainsi que Sanata Diabaté et les Professeurs Fabien Durif, Guy
Cucumel de l'Université du Québec à Montréal, Laure Ambroise de l'Université de Lyon 2 et Rémi Mencarelli de l'Université Savoie Mont-Blanc pour l'ensemble de leurs conseils.

\section{ORCID iD}

Isabelle Prim-Allaz (iD https://orcid.org/0000-00024620-5082

\section{Notes}

1. https://www.amisdelaterre.org/nouveau-rapportObsolescence-des.html

2. "I define Customer Value as an interactive relativistic preference experience». «By interactive, I mean that $\mathrm{C}$ in contrast to the position advocated by extreme subjectivists or extreme objectivists $\mathrm{C}$ Customer Value entails an interaction between some subject (a consumer) and some object (a product). » «By relativistic, I mean that Customer Value is (a) comparative (among objects), (b) personal (across people), and (c) situational (specific to the context) » in Holbrook (1996 : 138).

3. «Les modérateurs purs renvoient à la définition purement psychométrique de la notion de modération, selon laquelle un modérateur est une variable qui interagit avec la ou les variable(s) indépendantes mais n'est associée avec la variable dépendante tout au plus que de manière négligeable. Il n'y a donc pas d'effet « simple » du modérateur $[\mathrm{Z}]$ sur $\mathrm{Y}$ dans l'équation $\mathrm{Y}$ $=\mathrm{a}+\mathrm{bX}+\mathrm{c}(\mathrm{A} \times \mathrm{Z})+$ erreur. Cette dernière condition n'est pas exigée pour les quasi-modérateurs " in Caceres et Vanhame (2003 : 72). Un quasi-modérateur peut donc avoir simultanément un effet direct sur la variable dépendante et un effet modérateur.

4. https://www.insee.fr/fr/statistiques/2569368?somm aire $=2587886$, janvier 2019. Pourcentages calculés sur les actifs occupés de 15 ans ou plus vivant en France dans un ménage ordinaire.

https://laboratoire.agencedunumerique. gouv.fr/wp-content/uploads/sites/2/2017/11/ Baromc3a8tre20du20Numc3a9rique20-Prc3a9sentatio n20conf20de20presse2027nov2017.pdf, janvier 2019.

5. https://www.ecologique-solidaire.gouv.fr/feuilleroute-economie-circulaire-mieux-consommer-fairedurer-nos-produits, Avril 2018.

6. https://www.ecologique-solidaire.gouv.fr/ leconomie-circulaire, Avril 2018.

7. Le cabinet d'étude, en charge de la collecte des données, travaille à partir de la méthode des quotas sans redressement statistique. L'échantillon est représentatif des 18-65 ans sur les critères du 
sexe, de l'âge, de la CSP et de la segmentation géographique (5 zones) selon les données INSEE de janvier 2017.

8. Sur les 1270 répondants de l'échantillon principal, 24 d'entre eux soit $1,9 \%$ ont jeté leur téléphone à la poubelle. Ce pourcentage est relativement stable au fil des études (Kréziak et al., 2015).

9. https://www.ecologique-solidaire.gouv.fr/feuilleroute-economie-circulaire-mieux-consommer-fairedurer-nos-produits, Avril 2018.

10. Cette modalité a été considérée comme un comportement de garder. Kréziak et al. (2016) montrent, en effet, que certains individus donnent leur ancien téléphone comme jouet à leurs propres enfants et que, de fait, ce dernier ne quitte pas le foyer.

11. Une étude exploratoire menée par Kréziak et al. (2016) a montré que le recyclage renvoie, dans l'esprit des répondants à un dépôt dans un lieu où l'appareil part pour être « démembré » et valorisé, essentiellement en déchetterie.

12. Le téléphone peut avoir été fourni par l'employeur. Dans ce cas, soit il est rendu à l'employeur et c'est ce dernier qui définit la destinée, soit il est réapproprié par l'individu et l'on se retrouve dans une situation comparable à un téléphone personnel quant au choix de sa destinée.

13. Ces modalités sont conservées dans les traitements relatifs à « garder» versus « ne pas garder», mais ne sont pas prises en compte lorsque le choix de filière est analysé en détail.

14. https://www.ecologique-solidaire.gouv.fr/dechetsdequipements-electriques-et-electroniques, Janvier 2019.

15. Dans ce dernier cas, un modèle personnalisé a été retenu avec les 3 dimensions de la VRP introduit systématiquement dans l'analyse en méthode ascendante, l'ensemble des autres termes (TTG et effets d'interaction) étant introduits étape par étape. A nouveau, ce choix permet d'obtenir des tableaux de résultats ne présentant que les effets significatifs (sauf pour la VRP).

\section{Références bibliographiques}

ADEME (2016) Déchets, chiffres-clés. Disponible en ligne: https://www.ademe.fr/sites/default/files/assets/ documents/dechets-chiffres-cles-edition-2016-8813. pdf

ADEME (2017) Des tiroirs pleins de téléphones remplacés, consommateurs et objets à obsolescence programmée. Disponible en ligne: http://www.ademe.fr/ tiroirs-pleins-telephones-remplaces-consommateursobjets-a-obsolescence-percue.
Akaike H (1973) Information theory and an extension of the maximum likelihood principle. In: Petrov BN and Csaki . (éds), Second International Symposium on Information Theory, 267-281. Budapest: Academiai Kiado.

Albinsson P et Perera YB (2009) From trash to treasure and beyond: the meaning of voluntary disposition. Journal of Consumer Behavior 8(6): 340-343.

Ambroise L, Prim-Allaz I et Teyssier C (2018) Financial performance of servitized manufacturing firms: a configuration issue between servitization strategies and customer-oriented organizational design. Industrial Marketing Management 71(May): 54-68.

Bagozzi RP (1975) Marketing as exchange. Journal of Marketing 39(October): 32-39.

Baines TS, Lightfoot HW, Evans S, Neely A, Greenough R, Peppard J et Alcock JR (2007) State-of-theart in product-service systems. Proceedings of the Institution of Mechanical Engineers, Part B. Journal of Engineering Manufacture 221(10): 1543-1552.

Ballantine PW et Creery S (2010) The consumption and disposition behaviour of voluntary simplifiers. Journal of Consumer Behaviour 9(1): 45-56.

Beauvois JL et Joule R (1981) Soumission et idéologies: psychosociologie de la rationalisation. Paris: Presses Universitaires de France.

Belk RW (2013) Extended self in a digital world. Journal of Consumer Research 40(3): 477-500.

Bemmaor AC (1995) Predicting behavior from intention-to-buy measures: the parametric case. Journal of Marketing Research 32(2): 176-191.

Bergadaà M (2006) Le don d'objet: l'exploration de ses dimensions et des profils de donneurs aux œuvres de bienfaisance. Recherche et Applications en Marketing 21(1): 19-39.

Bianchi C et Birtwistle G (2010) Sell, give away, or donate: an exploratory study of fashion clothing disposal behaviour in two countries. The International Review of Retail, Distribution and Consumer Research 20(3): 353-368.

Birtwistle G et Moore CM (2007) Fashion clothing where does it all end up? International Journal of Retail \& Distribution Management 35(3): 210-216.

Blandin M-C (2016) 100 millions de téléphones portables usagés : l'urgence d'une stratégie, Rapport d'information - Sénat. Disponible en ligne: https://www. senat.fr/notice-rapport/2015/r15-850-notice.html

Bolton L E et Alba J (2012) When less is more: consumer aversion to unused utility. Journal of Consumer Psychology 22(3): 369-383.

Bowlby J. (1979). The making and breaking of affectional bonds. New York: Tavistock. 
Brough AR et Isaac MS (2012) Finding a home for products we love: how buyer usage intent affects the pricing of used goods. Journal of Marketing 76(4): 78-91.

Brezet J et van Hemel CG (1997). Ecodesign: A Promising Approach to Sustainable Production and Consumption. UNEP Ecodesign Manual Paris: UNEP.

Buckley PJ (2009) The impact of the global factory on economic development. Journal of World Business 44(2): 131-143.

Caceres RC et Vanhamme J (2003) Les processus modérateurs et médiateurs : distinction conceptuelle, aspects analytiques et illustrations. Recherche et Applications en Marketing 18(2): 67-100.

Carrington MJ, Neville BA et Whitwell GJ (2010) Why ethical consumers don't walk their talk: towards a framework for understanding the gap between the ethical purchase intentions and actual buying behaviour of ethically minded consumers. Journal of Business Ethics 97(1): 139-158.

Carson R (1997) Contingent evaluation surveys and tests of intensitivity to scope. Journal of Marketing Channels 12(3): 105-123.

Chandon P, Morwitz VG et Reinartz WJ (2005) Do intentions really predict behavior? Self-generated validity effects in survey research. Journal of Marketing 69(2): $1-14$.

Cherrier H (2009) Anti-consumption discourses and consumer-resistant identities. Journal of Business Research 62(2): 181-19.

Cherrier H et Murray JB (2007) Reflexive dispossession and the self: constructing a processual theory of identity. Consumption Markets \& Culture 10(1): 1-29.

Cherrier H et Ponnor T (2010) A study of hoarding behavior and attachment to material possessions. Qualitative Market Research: An International Journal 13(1): 8-23.

Chin WW (2010) How to write up and report PLS analyses. In: Vinzi VE, Chin WW, Henseler J et Wang H (éds) Handbook of Partial Least Squares, Concepts, Methods and Applications. Heidelberg: Springer Verlag, 655-690.

Cooper T (2004) Inadequate life? Evidence of consumer attitudes to product obsolescence. Journal of Consumer Policy 27(4): 421-449.

Coulter R et Ligas M (2003) To retain or to relinquish: exploring the disposition practices of packrats and purgers. Advances in Consumer Research 30: 38-43.

Cova V et Kréziak D (2013) Des riens aux biens : les pratiques de récup'création. Perspectives Culturelles de la Consommation 3(1): 47-78.

Cova V et Kréziak D (2016) La culture « Makers », une réponse des consommateurs à l'obsolescence program- mée à l'heure de l'économie circulaire. In: Lazzeri Y, Bonnet-Fernandez LYD et Domeizel M (éds) Economie circulaire et territoires :. Aix en Provence: Presses Universitaires d'Aix-Marseille, 161-174.

Cruz-Cárdenas J, González R et del Val Núñez MT (2015) The use of disliked gifts from a consumer behavior perspective. Journal of Business Research 68(7): 1635-1637.

De Certeau M (1980 [1990]) L'invention du quotidien, l'Arts de faire. Coll. Folio essais, Paris: Gallimard.

De Ferran F et Robinot E (2012) De l'identification des déterminants psychologiques de se débarrasser des objets aux comportements associés. 28ème Congrès International de l'AFM, Brest, 9-10 Mai 2012.

De Ferran F et Robinot E (2013) What makes people not throw goods away when they want to get rid of them? An application to books, clothes and mobile phones. Colloque EMAC, Istanbul, 4-7 Juin 2013.

Denegri-Knott J et Molesworth P (2009) I'll sell this and I'll buy them that: eBay and the management of possessions as stock. Journal of Consumer Behavior 8(6): 305-315.

Desjeux D, Monjaret A et Taponier S (1998) Quand les Français déménagent : circulation des objets domestiques et rituels de mobilité dans la vie quotidienne en France. Paris: Presses universitaires de France.

Domina T et Koch K (1999) Consumer reuse and recycling of post-consumer textile waste. Journal of Fashion Marketing and Management 3(4): 346-359.

Douglas M (1967) De la souillure : essai sur la notion de pollution et de tabou. Paris: La Découverte-Syros.

Ertimur B, Muñoz C, et Hutton JG (2015) Regifting: a multi-perspective processual overview. Journal of Business Research 68(9): 1997-2004.

Ertz M, Durif F, et Arcand M (2017) Life after death? Study of goods multiple lives practices. Journal of Consumer Marketing 34(2): 108-118.

Fornell C et Larcker DF (1981) Structural equation models with unobservable variables and measurement error: algebra and statistics. Journal of Marketing Research 18(3): 382-388.

Gregson N et Crewe L (2003) Second-hand cultures. Oxford et New York: Berg Publishers.

Gregson N, Metcalfe A et Crewe L (2007) Moving things along: the conduits and practices of divestment in consumption. Transactions of the Institute of British Geographers 32(2): 187-200.

Guillard V et Pinson C (2012) Comprendre et identifier les consommateurs qui ont tendance à «tout » garder. Recherche et Applications en Marketing 27(2): 57-79.

Ha-Brookshire JE et Hodges NN (2009) Socially responsible consumer behavior? Exploring usedclothing 
donation behaviour. Clothing and Textiles Research Journal 27(3) 179-196

Hair JF, Hult GTM, Ringle CM et Sarstedt M (2016) A Primer on Partial Least Squares Structural Equation Modeling (PLS-SEM) (2ème edn). Thousand Oaks, CA: SAGE.

Haws K, Reczek RW, Coulter RA et Bearden WO (2012) Keeping it all without being buried alive: understanding product retention tendency. Journal of Consumer Psychology 22: 224-236.

Helm R et Conrad D (2015) The impact of customer-specific and market-related variables on the preference for highly innovative products. Review of Managerial Science 9(1): 61-88.

Hibbert S, Horne S et Tagg S (2005) Charity retailers in competition for merchandise: examining how consumers dispose of used goods. Journal of Business Research 58(6): 819-828.

Hirschman EC, Ruvio A et Belk RW (2012) Exploring space and place in marketing research: excavating the garage. Marketing Theory 12(4): 369-389.

Holbrook MB (1996) Special session summary - customer value: a framework for analysis and research. Advances in Consumer Research 23: 138-142.

Holbrook MB (1999) Consumer Value: A Framework for Analysis and Research. New York, NY: Routledge.

Holbrook MB et Hirschman EC (1982) The experiential aspects of consumption: consumer fantasies, feelings, and fun. Journal of Consumer Research 9(2): 132-140.

Jacoby J, Berning CK et Dietvorst TF (1977) What about disposition? Journal of Marketing 41(April): 22-28.

Kahneman D, Knetsch JL et Thaler RH (1991) Anomalies: the endowment effect, loss aversion, and status quo bias. Journal of Economic Perspectives 5(1): 193-206.

Karababa E et Kjeldgaard D (2014) Value in marketing: toward sociocultural perspectives. Marketing Theory 14(1): 119-127.

Kates SM (2001) Disposition of possessions among families of people living with AIDS. Psychology \& Marketing 18 (4): 365-387.

Kleine S, Schutz R, Kleine III E et Allen CT (1995) How is a possession 'Me' or 'Not Me'? Characterizing types and an antecedent of material possession attachment. Journal of Consumer Research 22: 327-43.

Kozinets RV (2002) Can consumers escape the market? Emancipatory illuminations from Burning Man. Journal of Consumer Research 29(1): 20-38

Kréziak D, Prim-Allaz I, Robinot E et Durif F (2015) De la valeur d'usage à la valeur résiduelle : obsolescence perçue et destinée d'un produit remplacé. Congrès International de l'Association Française du Marketing, Marrakech, Mai.
Kréziak D, Prim-Allaz I, Robinot E et Durif F (2016) Obsolescence perçue, décision de renouveler et destinée des produits : le cas du téléphone portable. Décisions Marketing 81: 41-59.

Kumar V et Reinartz W (2016) Creating enduring customer value. Journal of Marketing 80(6) : 36-68.

Lastovicka JL et Fernandez KV (2005) Three paths to disposition: the movement of meaningful possessions to strangers. Journal of Consumer Research 31(4): 813-823.

Manski CF (1990) The use of intentions data to predict behavior: a best-case analysis. Journal of the American Statistical Association 85(412): 934-940.

McAlexander JH (1991) Divorce, the disposition of the relationship, and everything. Advances in Consumer Research 18: 43-48.

McCracken G (1988) Culture and Consumption. Bloomington and Indianapolis: Indiana University.

Monnot E, Reniou F et Rouquet A (2014) Le tri des déchets ménagers: une caractérisation des logistiques déployées par les consommateurs. Recherche et Applications en Marketing 29(3): 74-98.

Monnot E et Roux D (2017) Pannes des produits et obsolescence subie : une approche par les compétences des consommateurs. Congrès International de l'Association Française du Marketing, Tours, Mai.

Morgan LR et Birtwistle G (2009) An investigation of young fashion consumers' disposal habits. International Journal of Consumer Studies 33(2): 190-198.

Okada E (2001) Trade-ins, mental accounting and product replacement decisions. Journal of Consumer Research 27(4): 433-446.

Parasuraman A et Grewal D (2000) The impact of technology on the quality-value-loyalty chain. Journal of the Academy of Marketing Science 28(1): 168-174.

Parsons L (2008) Thomsons' rubbish theory: exploring the practices of value creation. European Advances in Consumer Research 8: 390-393.

Pavia T (1993) Dispossession and perceptions of self in late stage HIV infection. Advances in Consumer Research 20: 425-428.

Penalosa L et Mish J (2011) The nature and processes of market co-creation in triple bottom line firms: leveraging insights from consumer culture theory and service dominant logic. Marketing Theory 11(1): 9-34.

Phillips BJ et Sego T (2011) The role of identity in disposal: lessons from mothers' disposal of children's possessions. Marketing Theory 11(4): 435-454.

Pieters R (1991) Changing garbage disposition patterns. Journal of Public Policy and Marketing 10(2): $59-76$. 
Price LL, Arnould EJ et Folkman C (2000) Older consumers' disposition of special possessions. Journal of Consumer Research 27(2): 179-201.

Rivière A et Mencarelli R (2012) Vers une clarification théorique de la notion de valeur perçue en marketing. Recherche et Applications en Marketing 27(3): 97-123.

Robinot E et Trespeuch L (2016) De la seconde vie d'un objet aux émotions associées : une approche par la netnographie. Congrès de l'Association Française du Marketing, Lyon, mai.

Robinot E, Durif F, Kréziak D et Prim-Allaz I (2014) "Fifty ways to leave your cell phone": product obsolescence, product replacement and product attachment. EMAC, Valencia, Espagne, 3-6 Juin 2014.

Roster CA (2001) Letting go: the process and meaning of dispossession in the lives of consumers. Advances in Consumer Reasearch 28: 425-430.

Roux D et Guillard V (2016) Circulations d'objets entre étrangers dans l'espace public : une analyse des formes de socialité entre déposeurs et glaneurs. Recherche et Applications en Marketing 31(4): 30-49.

Roux D et Guiot D (2008) Une mesure des motivations envers l'achat d'occasion, leurs antécédents et leurs conséquences. Recherche et Applications en Marketing 23(4): 63-95.

Schifferstein HN et Zwartkruis-Pelgrim EP (2008) Consumer-product attachment: measurement and design implications. International Journal of Design 2(3): 1-13.

Strazzieri A (1994) Mesurer l'implication durable vis-à-vis d'un produit indépendamment du risque perçu. Recherche et Applications en Marketing 9(1): 73-91.

Thaler RH et Sunstein CR (2008) Nudge: improving decisions about health, wealth, and happiness. Constitutional Political Economy 19(4): 356-360.
Thompson M (1979) Rubbish Theory: The Vreation and Destruction of Value. Oxford: Oxford University Press.

Trudel R, Argo JJ et Meng MD (2016) The recycled self: consumers' disposal decisions of identity-linked products. Journal of Consumer Research 43(2): 246-264.

Türe M (2014) Value-in-disposition: exploring how consumers derive value from disposition of possessions. Marketing Theory 14(1): 53-72.

Urien B et Kilbourne W (2011) Generativity and selfenhancement values in eco-friendly behavioral intentions and environmentally responsible consumption behavior. Psychology \& Marketing 28(1): 69-90.

Venkatesh A et Peñaloza L (2014) The value of value in CCT. Marketing Theory 14(1): 135-138.

Webb TL et Sheeran P (2006) Does changing behavioral intentions engender behavior change? A meta-analysis of the experimental evidence. Psychological Bulletin 132(2): 249.

Wilson M (2016) When creative consumers go green: understanding consumer upcycling. Journal of Product \& Brand Management 25(4): 394-399.

Winterich KP, Reczek RW et Irwin JR (2017) Keeping the memory but not the possession: memory preservation mitigates identity loss from product disposition. Journal of Marketing 81(5): 104-120.

Woodall T (2003) Conceptualising 'Value for the consumer': an attributional, structiural and dispositional analysis. Academy of Marketing Science Review 12(1): 1-42.

Young MM et Wallendorf M (1989) Ashes to ashes, dust to dust: conceptualizing consumer disposition of possessions. Proceedings of the AMA winter educator's conference 33-39.

Zeithaml VA (1988) Consumer perceptions of price, quality, and value: a means-end model and synthesis of evidence. Journal of Marketing 52(3): 2-22. 


\section{Annexe I : Développement de l'échelle de valeur résiduelle perçue}

Tableau 7. ACP - Pré-test de l'échelle de valeur résiduelle, Rotation Promax (Echantillon étudiants, $\mathrm{n}=|5|-$ Téléphones portables).

\begin{tabular}{|c|c|c|c|}
\hline & \multicolumn{3}{|c|}{ Dimensions } \\
\hline & VRP utilitaire & \multicolumn{2}{|c|}{ VRP financière VRP affective } \\
\hline Mon téléphone pouvait encore me servir. & ,948 & & \\
\hline Mon téléphone pouvait être réutilisé. & ,913 & & \\
\hline Mon téléphone pouvait encore servir à quelqu'un. & ,872 & 127 & \\
\hline Mon téléphone avait une valeur de revente. & & 960 & \\
\hline $\begin{array}{l}\text { Mon téléphone valait encore quelque chose sur le plan } \\
\text { financier. }\end{array}$ & & ,947 & ,114 \\
\hline $\begin{array}{l}\text { Mon téléphone avait une valeur de reprise chez un } \\
\text { opérateur. }\end{array}$ & 196 & ,715 &,- 121 \\
\hline Je me sentais attaché(e) à mon téléphone. & & & ,941 \\
\hline Mon téléphone avait une valeur sentimentale. & & & 929 \\
\hline Mon téléphone est le témoin d'une époque. & 242 &,- 214 & ,605 \\
\hline$\%$ de variance: Totale $=80,870 \%$ & 44,232 & 23,734 & 12,904 \\
\hline Alpha de Cronbach & ,930 & 889 &, 782 \\
\hline \multicolumn{4}{|c|}{ Matrice des corrélations des composantes } \\
\hline VRP utilitaire & 1,000 & & \\
\hline VRP financière &, 500 & 1,000 & \\
\hline VRP affective & 135 & ,036 & 1,000 \\
\hline
\end{tabular}

Tableau 8. Valeur résiduelle perçue : Analyse en composante principale, Rotation Promax (Echantillon adultes, $n=$ 35 I - Téléphones portables).

\begin{tabular}{|c|c|c|c|}
\hline & \multicolumn{3}{|c|}{ Dimensions } \\
\hline & VRP utilitaire & VRP financière & VRP affective \\
\hline Mon téléphone pouvait encore servir à quelqu'un. & ,916 & & \\
\hline Mon téléphone pouvait encore me servir. & ,913 & & \\
\hline Mon téléphone pouvait être réutilisé. & ,880 & 104 & \\
\hline $\begin{array}{l}\text { Mon téléphone avait une valeur de reprise chez un } \\
\text { opérateur. }\end{array}$ & & 911 & \\
\hline Mon téléphone avait une valeur de revente. & ,124 & ,873 & \\
\hline $\begin{array}{l}\text { Mon téléphone valait encore quelque chose sur le plan } \\
\text { financier. }\end{array}$ & 168 & ,832 & \\
\hline Mon téléphone avait une valeur sentimentale. &,- 131 & & ,897 \\
\hline Je me sentais attaché(e) à mon téléphone. &,- 105 &, 134 & ,886 \\
\hline Mon téléphone est le témoin d'une époque. & ,345 &,- 308 &, 650 \\
\hline$\%$ de variance: Totale $=81,292 \%$ & 47,22 & 22,24 & 11,83 \\
\hline Alpha de Cronbach & 0,938 & 0,914 & 0,747 \\
\hline \multicolumn{4}{|c|}{ Matrice des corrélations des composantes } \\
\hline VRP utilitaire & 1,000 & & \\
\hline VRP financière &, 538 & 1,000 & \\
\hline VRP affective & ,063 &, 036 & 1,000 \\
\hline
\end{tabular}


Tableau 9. Valeur résiduelle perçue : Analyse en composante principale, Rotation Promax (Echantillon adultes, $\mathrm{n}=210-$ Sèche-cheveux).

\begin{tabular}{|c|c|c|c|}
\hline & \multicolumn{3}{|c|}{ Dimensions } \\
\hline & VRP financière & VRP utilitaire & VRP affective \\
\hline Mon sèche-cheveux pouvait encore me servir. & ,973 & & \\
\hline $\begin{array}{l}\text { Mon sèche-cheveux pouvait encore servir à } \\
\text { quelqu'un. }\end{array}$ & ,963 & & \\
\hline Mon sèche-cheveux pouvait être réutilisé. & ,940 & & \\
\hline $\begin{array}{l}\text { Mon sèche-cheveux avait une valeur de reprise } \\
\text { chez un distributeur. }\end{array}$ &,- 136 & ,962 & \\
\hline Mon sèche-cheveux avait une valeur de revente. & & ,895 & \\
\hline $\begin{array}{l}\text { Mon sèche-cheveux valait encore quelque chose } \\
\text { sur le plan financier. }\end{array}$ & 171 & ,834 & \\
\hline Mon appareil avait une valeur sentimentale. & & 113 & ,866 \\
\hline Je me sentais attaché(e) à mon sèche-cheveux. & & & ,864 \\
\hline Mon sèche-cheveux est le témoin d'une époque. & &,- 116 & ,749 \\
\hline$\%$ de variance: Totale $=82,491 \%$ & 49,35 & 21,60 & 11,54 \\
\hline Alpha de Cronbach &, 963 &, 904 &, 751 \\
\hline \multicolumn{4}{|c|}{ Matrice des corrélations des composantes } \\
\hline VRP financière & 1,000 & & \\
\hline VRP utilitaire &, 565 & 1,000 & \\
\hline VRP affective & 127 & ,275 & 1,000 \\
\hline
\end{tabular}

\section{Annexe 2 : Analyses confirmatoires - échelles de mesure, échantillon final}

Tableau 10. Echelle de mesure de la valeur résiduelle perçue - Analyse confirmatoire, Echantillon adultes, $n=$ 1302.

\begin{tabular}{|c|c|c|c|}
\hline & \multirow[t]{2}{*}{ Loadings } & \multicolumn{2}{|c|}{ Intervalle de confiance (95\%) } \\
\hline & & Borne inf. & Borne sup. \\
\hline \multicolumn{4}{|l|}{$\underline{\text { VRP utilitaire }}$} \\
\hline alpha Cronbach & & & \\
\hline Mon téléphone pouvait encore servir à quelqu'un & 954 & 945 & 962 \\
\hline Mon téléphone pouvait être réutilisé & ,954 & ,945 & ,962 \\
\hline Mon téléphone pouvait encore me servir & 934 & 923 & 944 \\
\hline \multicolumn{4}{|l|}{ VRP financière } \\
\hline alpha Cronbach & & & \\
\hline Mon téléphone avait une valeur de revente & 938 & 929 & 946 \\
\hline Mon téléphone valait encore quelque chose sur le plan financier & ,938 & ,929 & 947 \\
\hline Mon téléphone avait une valeur de reprise chez un opérateur & ,882 & ,863 & ,899 \\
\hline \multicolumn{4}{|l|}{ VRP affective } \\
\hline alpha Cronbach & & & \\
\hline Mon téléphone avait une valeur sentimentale & ,885 & ,855 & 907 \\
\hline Je me sentais attaché(e) à mon téléphone & ,898 & ,871 & 927 \\
\hline Mon téléphone est le témoin d'une époque & ,707 &, 620 & ,765 \\
\hline Echelle VRP & GoF & GoF (boostrap) & \\
\hline Absolu & ,801 & 8,800 & \\
\hline Relatif & 999 & 998 & \\
\hline Modèle externe & ,999 & ,998 & \\
\hline
\end{tabular}


Tableau I I. Echelle de mesure de la TTG - Analyse confirmatoire, Echantillon adultes, $n=1302$.

\begin{tabular}{|c|c|c|c|}
\hline & \multirow[t]{2}{*}{ Loadings } & \multicolumn{2}{|c|}{ Intervalle de confiance (95\%) } \\
\hline & & Borne inf. & Borne sup. \\
\hline \multicolumn{4}{|l|}{ TTG Sentimentale } \\
\hline alpha Cronbach $=, 95$ & & & \\
\hline Que de souvenirs de mon passé ! & ,950 & 941 & 959 \\
\hline Ça me rappelle tellement de choses & 961 & 954 & ,967 \\
\hline Ça me remémore tout ce que j'ai fait & ,950 & 940 & 959 \\
\hline \multicolumn{4}{|l|}{ TTG Sociale } \\
\hline alpha Cronbach $=, 91$ & & & \\
\hline Il doit bien y avoir quelqu'un à qui ça pourrait servir & ,898 & 879 & 915 \\
\hline Ça va sûrement intéresser quelqu'un un jour & 941 & ,932 & ,949 \\
\hline Un jour ou l'autre, je vais bien trouver quelqu'un qui va le(s) vouloir & ,925 & 913 & 935 \\
\hline \multicolumn{4}{|l|}{ ITG Economique } \\
\hline alpha Cronbach $=, 88$ & & & \\
\hline J'aurais l'impression de "jeter l'argent par les fenêtres" & 897 & 881 & 911 \\
\hline Ça représente de l'argent, je ne peux pas m'en débarrasser & ,906 & ,894 & ,918 \\
\hline Quand je pense au prix, ça me fait mal au cœur de m'en débarrasser & 901 & ,886 & 914 \\
\hline \multicolumn{4}{|l|}{ ITG Instrumentale } \\
\hline alpha Cronbach $=, 89$ & & & \\
\hline Je peux en avoir besoin un jour & ,937 & ,927 & 945 \\
\hline Ça peut toujours servir & 894 & 875 & ,910 \\
\hline Qui sait ? je peux avoir envie de m'en resservir plus tard & ,904 & ,890 & ,916 \\
\hline Echelle TTG & GoF & GoF (boostrap) & \\
\hline Absolu & ,809 & ,808 & \\
\hline Relatif & 999 & 999 & \\
\hline Modèle externe & ,999 & ,999 & \\
\hline
\end{tabular}

\section{Annexe 3 : Validités convergente et discriminante}

Tableau I2. Validités convergente et discriminante.

\begin{tabular}{|c|c|c|c|c|c|c|c|c|c|c|}
\hline Construit & Nbr d'items & $\alpha$ & CR & 1 & 2 & 3 & & & & 7 \\
\hline VRP utilitaire & 3 & 943 & 963 & $(, 898)$ & & & & & & \\
\hline VRP financière & 3 & 909 & ,943 & 340 & $(, 846)$ & & & & & \\
\hline VRP affective & 3 & 782 & 874 & 007 & ,002 & $(, 696)$ & & & & \\
\hline TTG Sentimentale & 3 & ,950 & ,968 & ,002 & , 010 & ,264 & $(, 910)$ & & & \\
\hline TTG Sociale & 3 & 911 & 944 & ,087 & 078 & 020 & ,094 & $(, 849)$ & & \\
\hline TTG Instrumentale & 3 & ,885 & ,929 & ,015 & ,053 &, 053 & , 142 & 104 & $(, 812)$ & \\
\hline TTG Economique & 3 & , 898 & ,936 & 070 & 018 & ,050 & 145 & 186 & ,234 & $(, 831)$ \\
\hline
\end{tabular}

